\title{
Plasma p-tau231: a new biomarker for incipient Alzheimer's disease pathology
}

\author{
Nicholas J. Ashton ${ }^{1,2,3,4}$. Tharick A. Pascoal ${ }^{5,6} \cdot$ Thomas K. Karikari $^{1} \cdot$ Andréa L. Benedet $^{1,5}$. \\ Juan Lantero-Rodriguez ${ }^{1} \cdot$ Gunnar Brinkmalm $^{1} \cdot$ Anniina Snellman $^{1} \cdot$ Michael Schöll ${ }^{1,2,10}$. Claire Troakes ${ }^{14}$. \\ Abdul Hye $^{3,4}$. Serge Gauthier ${ }^{7}$. Eugeen Vanmechelen ${ }^{8} \cdot$ Henrik Zetterberg $^{1,9,10,11}$. Pedro Rosa-Neto ${ }^{1,12,13}$. \\ Kaj Blennow ${ }^{1,9}$
}

Received: 3 December 2020 / Revised: 12 January 2021 / Accepted: 22 January 2021 / Published online: 14 February 2021

(c) The Author(s) 2021

\begin{abstract}
The quantification of phosphorylated tau in biofluids, either cerebrospinal fluid (CSF) or plasma, has shown great promise in detecting Alzheimer's disease (AD) pathophysiology. Tau phosphorylated at threonine 231 (p-tau231) is one such biomarker in CSF but its usefulness as a blood biomarker is currently unknown. Here, we developed an ultrasensitive Single molecule array (Simoa) for the quantification of plasma p-tau231 which was validated in four independent cohorts $(n=588)$ in different settings, including the full $\mathrm{AD}$ continuum and non-AD neurodegenerative disorders. Plasma p-tau 231 was able to identify patients with $\mathrm{AD}$ and differentiate them from amyloid- $\beta$ negative cognitively unimpaired (CU) older adults with high accuracy $(\mathrm{AUC}=0.92-0.94)$. Plasma p-tau231 also distinguished AD patients from patients with non-AD neurodegenerative disorders (AUC $=0.93$ ), as well as from amyloid- $\beta$ negative MCI patients $(\mathrm{AUC}=0.89)$. In a neuropathology cohort, plasma p-tau231 in samples taken on avergae 4.2 years prior to post-mortem very accurately identified $\mathrm{AD}$ neuropathology in comparison to non- $\mathrm{AD}$ neurodegenerative disorders ( $\mathrm{AUC}=0.99$ ), this is despite all patients being given an $\mathrm{AD}$ dementia diagnosis during life. Plasma p-tau231 was highly correlated with CSF p-tau231, tau pathology as assessed by $\left[{ }^{18} \mathrm{~F}\right] \mathrm{MK}-6240$ positron emission tomography (PET), and brain amyloidosis by $\left[{ }^{18} \mathrm{~F}\right]$ AZD469 PET. Remarkably, the inflection point of plasma p-tau231, increasing as a function of continuous $\left[{ }^{18} \mathrm{~F}\right]$ AZD469 amyloid- $\beta$ PET standardized uptake value ratio, was shown to be earlier than standard thresholds of amyloid- $\beta$ PET positivity and the increase of plasma $p$-tau181. Furthermore, plasma p-tau 231 was significantly increased in amyloid- $\beta$ PET quartiles $2-4$, whereas CSF p-tau 217 and plasma p-tau 181 increased only at quartiles 3-4 and 4, respectively. Finally, plasma p-tau231 differentiated individuals across the entire Braak stage spectrum, including Braak staging from Braak 0 through Braak I-II, which was not observed for plasma p-tau181. To conclude, this novel plasma p-tau231 assay identifies the clinical stages of AD and neuropathology equally well as plasma p-tau181, but increases earlier, already with subtle amyloid- $\beta$ deposition, prior to the threshold for amyloid- $\beta$ PET positivity has been attained, and also in response to early brain tau deposition. Thus, plasma p-tau231 is a promising novel biomarker of emerging AD pathology with the potential to facilitate clinical trials to identify vulnerable populations below PET threshold of amyloid- $\beta$ positivity or apparent entorhinal tau deposition.
\end{abstract}

Keywords Alzheimer's disease $\cdot$ Tau $\cdot$ Braak $\cdot$ Biomarkers $\cdot$ Blood $\cdot$ Preclinical $\cdot$ p-tau231 $\cdot$ p-tau181 $\cdot$ p-tau 217

Nicholas J. Ashton and Tharick A. Pascoal have contributed equally.

Pedro Rosa-Neto and Kaj Blennow have contributed equally as senior authors.

Nicholas J. Ashton

nicholas.ashton@gu.se

Kaj Blennow

kaj.blennow@neuro.gu.se

Extended author information available on the last page of the article

\section{Introduction}

The aberrant accumulation of aggregated amyloid- $\beta(\mathrm{A} \beta)$ peptides and abnormally phosphorylated tau protein as extracellular plaques and intraneuronal neurofibrillary tangles (NFTs), respectively, are the fundamental hallmarks of Alzheimer's disease (AD) neuropathology and remain to be the conclusive confirmation of the disease-thus a definitive diagnosis cannot be attributed until post-mortem. 
Nonetheless, the characterization of AD in vivo has been greatly enhanced by the visualization of $A \beta$ [52] and tau [35] aggregates by positron emission tomography (PET). The reduction of $A \beta 42$ or $A \beta 42 / 40$ and the increase of totaltau (t-tau) and phosphorylated tau (p-tau) represent the core cerebrospinal fluid (CSF) biomarker changes [43]. However, both CSF and PET biomarkers have significant practical, logistic and economical drawbacks and are unlikely to be widely used in primary care for the assessment of $\mathrm{AD}$ or other neurodegenerative diseases. Furthermore, given recent clinical trial failures, confirmatory evidence of underlying pathology is being more commonly required as a fundamental selection criterion for AD therapeutic trials. Therefore, the field would greatly benefit from a more easily implemented and cost-effective approach to the initial patient or participant assessment that could satisfy both clinical and drug development needs.

The emergence of blood biomarkers for the specific detection of AD pathophysiology offers the scalability required for patient or population triage. This would complement CSF and PET biomarkers in the initial assessment in memory clinics or therapeutic trial recruitment and monitoring. Blood neurofilament light (NfL), a biomarker of axonal injury, is robustly increased in $\mathrm{AD}[5,37]$ but is also increased in many other neurodegenerative disorders [4] and upon acute neurological injury [64]. Mass spectrometric assays for plasma $A \beta 42$ or $A \beta 42 / 40$ have demonstrated high accuracy in detecting cerebral $A \beta$ pathology $[45,56]$. However, the fold-change of A $\beta 42 / 40$ peptides, between $\mathrm{A} \beta$-positive and $\mathrm{A} \beta$-negative individuals, is substantially reduced in plasma than $\mathrm{CSF}$-likely owing to peripheral expression of $\mathrm{A} \beta$. Therefore, plasma NfL and $\mathrm{A} \beta$ do not have the required specificity to routinely detect underlying $\mathrm{AD}$ pathology. P-tau, however, is a highly specific pathological biomarker of $\mathrm{AD}$ and the diagnostic capabilities of CSF p-tau at threonine 181 (p-tau181) [12] have been widely replicated in blood. Plasma p-tau181 can differentiate AD from non-AD neurodegenerative disorders [10, 25, 27, 29, 34, 60], detect $\mathrm{AD}$ neuropathology $[25,34,60]$, identifies individuals with increased $A \beta$ and tau PET retention $[25,27,29,41$, $60]$, strongly associates with imminent grey matter atrophy [57] and predicts progression to AD dementia, to the same degree as CSF p-tau181 [25, 27, 44]. Furthermore, findings of CSF p-tau at threonine 217 (p-tau217), which suggests stronger associations with $\mathrm{AD}$ pathology than standard CSF p-tau 181 biomarkers $[6,26,28]$, have also been replicated in plasma $[38,48]$. Therefore, plasma p-tau 181 and p-tau 217 are highly promising clinical biomarkers in the assessment of memory complaints and risk of disease progression.

The presence of $\mathrm{p}$-tau in biofluids has been proposed to be a cellular response to NFT pathology [23]. However, the increases of CSF p-tau residues precede detectable NFT pathology, indexed by tau PET, by up to a decade $[8,62]$, and plasma p-tau181 is already increased in tau PET-negative but A $\beta$ PET-positive individuals [29]. This demonstrates that soluble tau, in CSF and plasma, is likely indicative of early tau pathology which is closely correlated with $A \beta$ deposition. We have recently described the increase of different phosphoforms of tau in CSF in preclinical and prodromal disease [3, 28, 58]. In two independent studies [3, 58], we found that CSF p-tau phosphorylated at threonine 231 (p-tau231) is a biomarker of very early tau pathology. CSF p-tau 231 begins to increase concurrently with $A \beta$ pathology in preclinical disease [58] and in $A \beta$-negative $C U$ individuals shows focal associations with emerging $A \beta$ pathology in the medial orbitofrontal, precuneus and posterior cingulate cortices [3]. To this end, given the successful translation of the CSF p-tau181 and CSF p-tau217 assays to blood tests, we hypothesized that the detection of p-tau 231 in plasma would be possible, and further, would reflect the advantageous characteristics of CSF p-tau231 being a biomarker for very early $\mathrm{AD}$ pathology, which begins to increase before A $\beta$ PET positivity is achieved. Such a biomarker could be utilized as a simple and cost-effective biomarker to detect preclinical or even earlier emerging pathology prior to substantial $A \beta$ accumulation for therapeutic trials recruitment and would be highly complementary to the characteristics of the plasma p-tau181 and p-tau217 markers.

In this study, we report the first ultrasensitive immunoassay for the precise quantification of plasma p-tau231. We assessed the capabilities of this novel blood biomarker in the detection of in vivo AD pathophysiology, specifically; (i) the differentiation of $\mathrm{AD}$ from cognitively healthy individuals and other neurodegenerative diseases, including neuropathological confirmation; (ii) the segregation of individuals across the AD continuum, (iii) use as a primary care biomarker and (iv) the determination of forthcoming cognitive decline and hippocampal atrophy. Further, given our recent findings for CSF p-tau231, we also assessed if plasma p-tau 231 could detect early abnormalities in amyloid- $\beta$ or tau PET scans, with a focus on preclinical disease and in comparison to p-tau181 and p-tau217 epitopes.

\section{Methods}

\section{Sample cohorts}

The discovery cohort included plasma from biochemically defined AD patients $(n=20)$ and age-matched controls $(n=18)$. The AD patients were clinically assessed for suspected $\mathrm{AD}$ and demonstrated no evidence of other neurological conditions (e.g., co-existing inflammatory or cerebrovascular disease). AD patients demonstrated a typical AD CSF biomarkers profile; CSF A $\beta 42<530 \mathrm{ng} / \mathrm{L}$, CSF p-tau $>60 \mathrm{ng} / \mathrm{L}$, and CSF t-tau $>350 \mathrm{ng} / \mathrm{L}$. The control 
group consisted of patients with minor neurological or psychiatric symptoms, and with core CSF biomarker levels within normal ranges. The use of these patient samples has been approved by the Ethics Committee at the University of Gothenburg (EPN 140811).

The discovery findings were independently replicated in the Translational Biomarkers of Aging and Dementia (TRIAD) cohort, McGill University, Canada [51] $(n=313)$. In the TRIAD cohort, participants had CSF and PET (amyloid- $\beta$ and tau) biomarkers and detailed clinical and cognitive assessments, including Mini-Mental State Examination (MMSE) and the clinical dementia rating (CDR) tests. The TRIAD cohort consisted of cognitively unimpaired (CU) young (age range, 20-30 years) and CU elderly (age range, 50-86 years) as well as mild cognitive impairment (MCI), AD, and non-AD dementia patients. CU participants $(n=16)$ who were considered middle-age adults (age range, 30-50 years) were included in the CU elderly group for the analysis. CU participants had an MMSE score $>24$ and a CDR score of 0 . MCI participants had a CDR score of 0.5 , subjective and objective impairments in cognition, but preserved activities of daily living. AD dementia patients had a CDR score $\geq 0.5$ and met the National Institute on Aging and the Alzheimer's Association criteria for probable Alzheimer's disease determined by a physician [40]. The non-AD dementia participants had CDR score $\geq 0.5$, were A $\beta$ PET negative and had a clinical diagnosis of FTD $(n=10)$, primary progressive aphasia $(n=1)$, cortical basal degeneration $(n=1)$, progressive supranuclear palsy $(n=2)$, vascular cognitive impairment $(n=10)$, hippocampal sclerosis $(n=1)$, or cerebral amyloid angiopathy $(n=1)$. In the TRIAD cohort, participants were excluded if they had active substance abuse or inadequately treated conditions, recent head trauma or major surgery, or if they presented safety contraindication for the study procedures.

In the primary care setting cohort $(n=190)$ we aimed to evaluate the new blood biomarker in the real-life setting, where blood biomarkers were likely to have a significant role. We included controls from the community without a diagnosis of a neurological condition and patients referred to the McGill University Research Centre for Studies in Aging memory clinic, Canada, from primary care physicians. These individuals had received a clinical diagnosis by the primary care physicians but were yet to undergo biomarker and clinical assessments in the specialized memory clinic setting. The TRIAD and the primary-care cohort were approved by the Douglas Mental Health University Institute Research Ethics Board and the Montreal Neurological Institute PET working committee, and a written informed consent was obtained for all participants.

The neuropathology cohort $(n=47)$ aimed to evaluate the specificity of p-tau231 in cases with a definite diagnosis of $\mathrm{AD}$ against other neurodegenerative disorders with a definite diagnosis (not "only" clinical evaluations and PET scans as for the validation and primary care cohorts). We included $\mathrm{AD}$ and non-AD neuropathologically confirmed cases who provided a plasma sample $1-9$ years (mean $=4.2$ years) prior to death. At the time of clinical assessment, a diagnosis of probable or possible $\mathrm{AD}$ (AD dementia) was made according to the Diagnostic and Statistical Manual for Mental Diagnosis, fourth edition and National Institute of Neurological, Communicative Disorders and Stroke-Alzheimer's disease and Related Disorders Association (NINCDS-ADRDA) clinical criteria [39]. No CSF or imaging assessments were performed for these individuals. Consent for autopsy, neuropathological assessment and research was obtained for all cases and the study was carried out under the ethical approval of the Medical Research Council (MRC) London Neurodegenerative Diseases Brain Bank, Institute of Psychiatry, King's College London. Block taking and neuropathological assessment was performed according to standard criteria for the diagnosis of neurodegenerative disease. Assessments included Braak staging for NFT [13] and reporting of co-existing pathology such as cerebrovascular lesions, TAR DNA-binding protein 43 (TDP-43), and Lewy body pathology. Non-AD dementias had no or sparse neurite plaque pathology and were classified as progressive supranuclear palsy $(n=3)$, frontotemporal lobe degermation $(n=3)$, Lewy body dementia $(n=2)$, vascular dementia $(n=2)$, cerebral amyloid angiopathy $(n=1)$ by two independent pathologists.

\section{Phosphorylated tau blood measurements}

All plasma p-tau biomarkers in this study were measured using in-house Single molecule array (Simoa) methods on the HD-X instrument (Quanterix) at the Clinical Neurochemistry Laboratory, Sahlgrenska University Hospital, Mölndal, Sweden. Plasma p-tau181 and plasma NfL measurements used in this study have been previously reported $[9,29]$.

For the novel plasma p-tau231 Simoa assay, monoclonal mouse antibodies were generated using a synthetic peptide $\left(\mathrm{K}_{224} \mathrm{KVAVVR} \mathrm{pT}\right)$ PPKSPSSAK $\left.{ }_{240} \mathrm{C}\right)$ as a KLH-coupled antigen, numbered according to full-length tau- 441 phosphorylated on threonine 231. Candidate hybridomas were selected on brain extracts of $\mathrm{AD}$ and control brain tissue. The final cloned and purified monoclonal antibody, ADx253, was characterized on synthetic peptides spanning amino acids threonine 217 till serine 241 of full-length tau for its affinity, its phospho-specificity using both phosphorylated and non-phosphorylated peptides and its preferred selectivity in which position 232 was replaced by a Pip, to simulate cis-selectivity of ADx253. A biotin-conjugated $\mathrm{N}$-terminal anti-tau mouse monoclonal antibody was used for detection. Full-length recombinant tau 441 phosphorylated in vitro by 
glycogen synthase kinase $3 \beta$ was used as the calibrator. The assay validation focused on dilution linearity, spike recovery, antibody specificity, precision and lower limit of quantification (LLOQ) are described in the supplementary methods, supplementary Table 1-3 and supplementary Fig. 1-2, online resource.

\section{CSF measurements}

In the discovery cohort, CSF p-tau181, total tau and A $\beta 42$ were measured using the established INNOTEST ELISA assays from Fujirebio. The fully automated LUMIPULSE G1200 (Fujirebio) was used to measure CSF p-tau181, total tau and $A \beta 42 / A \beta 40$ for the validation and clinical cohorts. CSF p-tau217 and CSF p-tau231 were quantified by the custom Simoa and ELISA assays, respectively, and have been previously described in detail $[28,58]$.

\section{Imaging analysis}

All individuals in the TRIAD cohort were assessed with Siemens 3 T MRI as well as A $\beta\left[{ }^{18} \mathrm{~F}\right]$ AZD4694 PET and tau $\left[{ }^{18} \mathrm{~F}\right] \mathrm{MK}-6240$ PET acquired with a Siemens HighResolution Research Tomograph. $\left[{ }^{18} \mathrm{~F}\right] \mathrm{MK}-6240$ images were acquired at $90-110 \mathrm{~min}$ after the intravenous bolus injection of the radiotracer $[50,51] .\left[{ }^{18} \mathrm{~F}\right] \mathrm{AZD} 4694$ PET images were acquired at 40-70 min after the intravenous bolus injection of the radiotracer [50, 51]. The PET images were spatially smoothed to achieve a final 8-mm full width at half maximum resolution and were processed using a previously described pipeline [50, 51], $\left[{ }^{18} \mathrm{~F}\right] \mathrm{MK} 6240$ images were stripped off the meninges before smoothing, as described elsewhere [51]. $\left[{ }^{18}\right.$ F]MK-6240 SUVR was measured regionally in the transentorhinal (stage I-II), limbic (III-IV), and isocortical (V-VI) regions approximating Braak stages of NFT pathology, as previously described, and tau positivity was defined as 2.5 standard deviations (SD) higher than the mean SUVR of CU A $\beta$-negative elderly [29]. Individuals negative for tau PET uptake in all aforementioned regionsof-interest were classified as Braak stage 0. Global $\left[{ }^{18} \mathrm{~F}\right]$ AZD4694 SUVR was derived from averaging retention in the precuneus, the cingulate, inferior parietal, medial prefrontal, lateral temporal, and orbitofrontal cortices. A $\left[{ }^{18} \mathrm{~F}\right]$ AZD4694 SUVR positivity was determined at SUVR $>1.55$ (centiloid [32] =22), as detailed elsewhere [59]. The intracranial volume corrected hippocampal volume was measured with Freesurfer version 6.0 using the Desikan-Killiany-Tourville gray matter parcellation [31].

\section{Statistical analysis}

The statistical analyses were performed using R statistical software version 3.1.2 (http://www.r-project.org/). The voxel-wise statistics were performed using MATLAB v9.2 using the VoxelStats package [36]. The group-wise comparisons were assessed using unpaired analysis of variance with Tukey's multiple comparisons test at $P<0.05$, whereas associations between biomarkers were tested with linear regressions or Spearman rank correlation analysis. Voxel-wise associations were performed with linear regression models false discovery rate corrected for multiple comparisons at $P<0.05$. The receiver operating characteristic curve (ROC) comparing groups provided the area under the curve (AUC) for a clinical diagnosis. In addition, we modeled and plotted p-tau biomarker levels as a function of $\mathrm{A} \beta$ PET load using a local weighted regression model, as performed elsewhere [17, 42, 47]. In this analysis, plasma p-tau 231 and p-tau 181 biomarker values were corrected for age and sex and converted to z-scores anchored on the normative data of $\mathrm{CU} A \beta$-negative. The associations between plasma p-tau231 concentrations and longitudinal changes in MMSE score and hippocampal volume were assessed with linear regression models. The longitudinal changes in MMSE score and hippocampal volume were calculated as the difference between followup and baseline values divided by time duration between the measurements.

\section{Results}

\section{Development and performance of plasma p-tau231 single-molecule array (Simoa)}

The plasma p-tau231 assay demonstrated high analytical performance (supplementary Table 1-2, online resource) with high precision within and between clinical studies (supplementary Table 3, online resource). Immunoprecipitation and mass spectrometric studies showed that the assay specifically measures $\mathrm{N}$-terminal to mid-domain forms of tau phosphorylated at threonine- 231 and does not recognize non-phosphorylated forms of tau (supplementary Fig. 1-2, online resource). For the determination of LLOQ, the calibrator was analysed in duplicates at concentrations between 64 and $0.25 \mathrm{pg} / \mathrm{mL}$. The deviation of the signal-calculated concentrations from the known values was estimated. The LLOQ was set as the calibrator 
point before the coefficient of variance increased above $20 \%$. In the clinical studies, 588 out of 597 (98.5\%) samples measured above the assay LLOQ of $2 \mathrm{pg} / \mathrm{ml}$. The 9 samples measuring below the LLOQ included A $\beta$-negative $\mathrm{CU}(n=6)$ and non-AD cases $(n=3)$.

\section{Study participants}

There were 38 individuals in the discovery cohort (supplementary table 4 , online resource). We assessed a further 313 individuals from the TRIAD cohort (McGill University,

Table 1 Characteristics of the TRIAD cohort

\begin{tabular}{|c|c|c|c|c|c|}
\hline & \multicolumn{5}{|l|}{$\begin{array}{l}\text { TRIAD cohort } \\
(n=313)\end{array}$} \\
\hline & Young adults $(n=32)$ & CU elderly adults $(n=159)$ & $\operatorname{MCI}(n=54)$ & $\mathrm{AD}(n=42)$ & $\begin{array}{l}\text { Non-AD } \\
(n=26)\end{array}$ \\
\hline Age, years & $22.8(1.5)^{\dagger}$ & $69.2(10.2)$ & $69.8(7.1)$ & $65.7(9.2)$ & $66.7(7.1)^{*}$ \\
\hline $\begin{array}{l}\text { Sex } \\
\text { Women } \\
\text { Men }\end{array}$ & $\begin{array}{l}20(62.5 \%) \\
12(37.5 \%)\end{array}$ & $\begin{array}{l}101(63.5 \%) \\
58(36.5 \%)\end{array}$ & $\begin{array}{l}29(53.7 \%) \\
25(46.3 \%)\end{array}$ & $\begin{array}{l}20(47.6 \%) \\
22(52.4 \%)\end{array}$ & $\begin{array}{l}12(46.1 \%) \\
14(45.1 \%)\end{array}$ \\
\hline$A P O E \& 4$ carriership & $8(25 \%)^{\dagger}$ & $45(28.3 \%)^{\dagger}$ & $27(50 \%)$ & $29(69.1 \%)^{*}$ & $5(19.2 \%)^{\dagger}$ \\
\hline MMSE score & $29.8(0.5)^{\dagger}$ & $29.1(1.1)^{\dagger}$ & $27.8(1.8)^{\dagger^{*}}$ & $18.5(5.7)^{*}$ & $25.6(9.7)^{\dagger}$ \\
\hline $\begin{array}{l}\text { CSF Biomarkers }(\mathrm{pg} / \mathrm{mL}) \\
\text { A } \beta 42 \\
\text { P-tau181 } \\
\text { T-tau }\end{array}$ & $\begin{array}{l}828.7(242.6)^{\dagger} \\
22.7(6.1)^{\dagger} \\
201.7(51.2)^{\dagger}\end{array}$ & $\begin{array}{l}986.0(432.2)^{\dagger} \\
43.3(40.2)^{\dagger} \\
333.3(122.2)^{\dagger}\end{array}$ & $\begin{array}{l}706.0(345.3)^{\dagger^{*}} \\
79.8(59.1)^{\dagger *} \\
502.9(295.7)^{\dagger^{*}}\end{array}$ & $\begin{array}{l}439.0(142.2)^{*} \\
106.6(39.6)^{*} \\
700.4(345.8)^{*}\end{array}$ & $\begin{array}{l}930.3(165.7)^{\dagger} \\
32.9(9.2)^{\dagger} \\
311.9(70.3)^{\dagger}\end{array}$ \\
\hline $\begin{array}{l}\text { A } \beta \text { PET } \\
\text { SUVR } \\
\% \text { A } \beta \text {-positive }\end{array}$ & $\begin{array}{l}1.15(0.2)^{\dagger} \\
0 \%\end{array}$ & $\begin{array}{l}1.38(0.4)^{\dagger} \\
19.4 \%\end{array}$ & $\begin{array}{l}1.96(0.6)^{\dagger} \\
79.6 \%\end{array}$ & $\begin{array}{l}2.19(0.5)^{*} \\
100 \%\end{array}$ & $\begin{array}{l}1.21(0.1)^{\dagger} \\
0 \%\end{array}$ \\
\hline $\begin{array}{l}\text { Tau PET SUVR } \\
\text { Braak I-II } \\
\text { Braak III-IV } \\
\text { Braak V-VI }\end{array}$ & $\begin{array}{l}0.85(0.3)^{\dagger} \\
0.91(0.9)^{\dagger} \\
1.1(0.2)^{\dagger}\end{array}$ & $\begin{array}{l}0.97(0.2)^{\dagger} \\
0.95(0.1)^{\dagger} \\
0.97(0.1)^{\dagger}\end{array}$ & $\begin{array}{l}1.58(0.6)^{\dagger^{*}} \\
1.29(0.5)^{\dagger^{*}} \\
1.14(0,5)^{\dagger^{*}}\end{array}$ & $\begin{array}{l}1.98(0.6){ }^{*} \\
2.61(1.1)^{*} \\
2.25(2.1)^{*}\end{array}$ & $\begin{array}{l}0.87(0.1)^{\dagger^{*}} \\
0.91(0.1)^{\dagger} \\
0.96(0.1)^{\dagger}\end{array}$ \\
\hline $\begin{array}{l}\text { Plasma biomarkers }(\mathrm{pg} / \mathrm{mL}) \\
\text { P-tau181 } \\
\text { P-tau231 } \\
\text { NfL }\end{array}$ & $\begin{array}{l}8.22(2.6)^{\dagger} \\
9.41(3.2)^{\dagger} \\
6.24(1.3)^{\dagger}\end{array}$ & $\begin{array}{l}10.91(3.3)^{\dagger} \\
14.94(4.1)^{\dagger} \\
23.12(2.2)^{\dagger}\end{array}$ & $\begin{array}{l}16.26(6.7)^{\dagger *} \\
19.45(7.1)^{\dagger *} \\
24.62(8.2)^{\dagger}\end{array}$ & $\begin{array}{l}25.21(7.8)^{*} \\
29.22(8.2)^{*} \\
34.04(8.3)^{*}\end{array}$ & $\begin{array}{l}9.91(2.1)^{\dagger} \\
11.53(4.1)^{\dagger} \\
28.31(7.2)^{*}\end{array}$ \\
\hline
\end{tabular}

Data in mean (SD) or $n(\%)$. We used analysis of variance followed by Tukey's post hoc test to assess differences between groups for continuous variables. For sex and $A P O E \varepsilon 4$ genotype, we used contingency $\chi^{2}$ tests. $C U=$ cognitively unimpaired. $\mathrm{MCI}=$ mild cognitive impairment. MMSE $=$ Mini-Mental State Examination. SUVR=standardised uptake value ratio. p-tau181=tau phosphorylated at threonine 181 . p-tau231 $=$ tau phosphorylated at threonine $231 . \mathrm{NfL}=$ neurofilament light chain. ${ }^{*} p<0.05$ compared with cognitively unimpaired older adults. $\dagger p<0.05$ compared with Alzheimer's disease

Table 2 Characteristics of the primary care and neuropathology cohort

\begin{tabular}{|c|c|c|c|c|c|c|}
\hline & \multicolumn{4}{|c|}{ Primary care cohort $(n=190)$} & \multicolumn{2}{|c|}{ Neuropathology $(n=47)$} \\
\hline & Young adults $(n=8)$ & $\begin{array}{l}\text { CU elderly } \\
\text { adults }(n=131)\end{array}$ & MCI $(n=17)$ & $\mathrm{AD}(n=34)$ & $\begin{array}{l}\mathrm{AD} \\
(n=36)\end{array}$ & Non-AD $(n=11)$ \\
\hline Age, years & $23.1(1.2)^{*}$ & $70.8(8.1)$ & $70.1(7.2)$ & $65.4(13.2)$ & $81.5(17.5)$ & $79.7(10.9)$ \\
\hline $\begin{array}{l}\text { Sex } \\
\text { Men } \\
\text { Women }\end{array}$ & $\begin{array}{l}5(62.5 \%) \\
3(37.5 \%)\end{array}$ & $\begin{array}{l}44(33.5 \%) \\
87(66.5 \%)\end{array}$ & $\begin{array}{l}6(35.3 \%) \\
11(64.7 \%)\end{array}$ & $\begin{array}{l}13(38.2 \%) \\
21(61.8 \%)\end{array}$ & $\begin{array}{l}12(33.3 \%) \\
24(66.7 \%)\end{array}$ & $\begin{array}{l}5(45.5 \%) \\
6(54.5 \%)\end{array}$ \\
\hline$A P O E \& 4$ carriership & $3(37.5 \%)$ & $31(23.6 \%)$ & $6(35.3 \%)$ & $20(58.8 \%$ & $20(55.6 \%)$ & $2(18.2 \%)$ \\
\hline Education, years & $18.8(2.4)$ & $14.1(3.6)$ & $13.0(4.5)$ & $8(4.1)^{\mathrm{a}}$ & $31.5(9.5)^{\mathrm{b}}$ & $13.2(2.7)$ \\
\hline Braak Score (I/II/III/IV/V/VI) & ... & $\ldots$ & ... & $\ldots$ & $0 / 0 / 0 / 7 / 5 / 24$ & $1 / 5 / 5 / 0 / 0 / 0$ \\
\hline $\begin{array}{l}\text { Time from plasma collection to } \\
\text { post-mortem, years (range) }\end{array}$ & ... & $\ldots$ & ... & $\ldots$ & $4.0(0.8-8)$ & $4.2(2-9)$ \\
\hline
\end{tabular}

${ }^{\mathrm{a}} p<0.01$ compared with cognitively unimpaired older adults

${ }^{\mathrm{b}} p<0.0001$ compared with non-AD 
Canada). This included young adults $(n=32)$, CU elderly $(n=189), \mathrm{MCI}(n=54), \mathrm{AD}(n=42)$ and non-AD neurodegenerative disorders $(n=26)$, which is displayed in Table 1 . The primary care cohort ( $n=190$, Table 2$)$ are individuals who have received a clinical diagnosis but were yet to undergo biomarker and clinical assessments in the specialized memory clinic setting. The neuropathology cohort ( $n=47$, Table 2$)$ included individuals with a plasma sample obtained between 1 and 9 years (mean $=4.2$ years) prior to post-mortem where a definite neuropathological diagnosis of either $\mathrm{AD}(n=36)$ or non-AD dementia $(n=11)$.

\section{Plasma p-tau231 shows high performance for the diagnosis of $A D$}

In the discovery cohort, plasma p-tau231 concentration showed high performance for the diagnosis of $\mathrm{AD}$ dementia (AUC $=0.94$ (95\% CI $=0.87-1.0)$, Fig. 1a, b). The addition of age, sex, and APOE $\varepsilon 4$ genotype in the models did not significantly increase the diagnostic performance of plasma p-tau231.

In the TRIAD cohort, plasma p-tau231 was significantly increased in AD dementia and MCI A $\beta$-positive groups compared to $\mathrm{A} \beta$-negative groups $(P<0.0001)$. Furthermore, plasma p-tau231 was significantly increased in CU elderly $\mathrm{A} \beta$-positive and MCI A $\beta$-positive compared to A $\beta$-negative groups $(P<0.0001$, Fig. $1 \mathrm{c}, \mathrm{d}$; $)$. The lower accuracy in distinguishing $\mathrm{AD}$ dementia from $\mathrm{CU}$ elderly $\mathrm{A} \beta$-positive $(\mathrm{AUC}=0.67)$ and MCI $\mathrm{A} \beta$-positive $(\mathrm{AUC}=0.72)$ compared with plasma p-tau181 (supplementary table 5, online resource) illustrated that plasma p-tau 231 has already increased at the preclinical phase of the disease. Plasma p-tau231 was highly accurate in detecting $\mathrm{AD}$ in all dementia cases $(\mathrm{AUC}=0.93)$. In comparison to plasma p-tau181, p-tau231 showed no significant difference in the prediction of $\mathrm{AD}$ dementia from non-AD dementias in the TRIAD cohort (supplementary Fig. 3, online resource); this remained true for all other comparisons between AD (supplementary table 5, online resource) and MCI A $\beta$-positive (supplementary table 6, online resource) with other groups included in the study. However, plasma p-tau231 was significantly better than plasma $\mathrm{p}$-tau 181 in distinguishing $\mathrm{CU}$ elderly $\mathrm{A} \beta$-positive from MCI A $\beta$-negative $(P<0.05$, supplementary table 7 , online resource), while the reverse was true in separating $\mathrm{CU}$ elderly $\mathrm{A} \beta$-positive from $\mathrm{AD}(P<0.05$, supplementary table 7 , online resource). In the whole group, plasma p-tau231 correlated with plasma p-tau181 $(r=0.654$, $P<0.0001$ ), which was stronger in A $\beta$-positive individuals $(r=0.611, P<0.0001)$ than $\mathrm{A} \beta$-negative individuals $(r=0.444, P<0.0001)$. Plasma $\mathrm{p}$-tau231 also correlated with plasma NfL $(r=0.414, P<0.0001)$.
In the primary care setting, plasma p-tau231 concentration increased progressively from CU young to elderly, MCI, and AD dementia patients $(P<0.0001$, Fig. 1e). Plasma p-tau231 discriminated $\mathrm{AD}$ dementia from $\mathrm{CU}$ young and elderly (AUC $=0.91$ and $\mathrm{AUC}=0.75$, Fig. 1f) but not from MCI (AUC=0.63) (Fig. 1f).

In the neuropathology cohort, plasma p-tau231 was confirmed as being highly accurate for discriminating between $\mathrm{AD}$ dementia and non-AD neurodegenerative $(\mathrm{AUC}=0.997,95 \% \mathrm{CI}=0.988-1.00$ ), Fig. $1 \mathrm{~h}$ ). Again, in this analysis, plasma p-tau231 was not significantly better than plasma p-tau181 (AUC $=0.9293,95 \%$ $\mathrm{CI}=0.8551-1.00)$ despite being numerically higher in the same patients. A stepwise increase of plasma p-tau231 between Braak I-II, III-IV and V-VI was observed ( $P=0.0004$, supplementary Fig. 4a, online resource) which was less pronounced for plasma p-tau181 $(P=0.0125$, supplementary Fig. $4 b$, online resource). Plasma p-tau 231 had a higher accuracy in discriminating Braak I-II and V-VI $(\mathrm{AUC}=0.99,95 \% \mathrm{CI}=0.97-1.00)$ and Braak I-II and Braak III-IV (AUC $=0.75,95 \% \mathrm{CI}=0.61-0.98)$ in comparison to p-tau181 (supplementary Fig. 4c, online resource). There was no noticeable difference when observing the concentration of p-tau231 in the specific diagnosis of non-AD neurodegenerative disorders for the neuropathology cohort or the validation cohort (supplementary table 9, online resource).

In all cohorts, the plasma p-tau231 levels were approximately twofold higher in $\mathrm{AD}$ than $\mathrm{CU}$ combined (threefold higher in $\mathrm{AD}$ than in $\mathrm{CU}$ elderly-A $\beta$-negative). Plasma p-tau231 was 2.6-fold higher in $\mathrm{AD}$ compared to non- $\mathrm{AD}$ dementias.

\section{Plasma p-tau231 is closely related to CSF and PET biomarkers}

In the validation cohort, plasma p-tau231 was strongly associated with tau $\left[{ }^{18} \mathrm{~F}\right] \mathrm{MK}-6240$ SUVR values across the brain cortex with the highest association in the temporal and cingulate cortices as well as with CSF p-tau231 $(P<0.0001$; Fig. 2a, b). Plasma p-tau231 also showed a strong correlation with $\mathrm{A} \beta\left[{ }^{18} \mathrm{~F}\right] \mathrm{AZD} 4694$ PET across the brain cortex with the highest association in the precuneus, frontal cortex, and striatum (Fig. 2c). Interestingly, in CU individuals, the relationship between plasma p-tau 231 and $\mathrm{A} \beta$ PET existed in both $\mathrm{A} \beta$-positive $(P<0.01, r=0.502)$ and $\mathrm{A} \beta$-negative $(P<0.0001, r=0.389)$ individuals. However, in cognitively impaired (CI) patients, plasma $\mathrm{p}$-tau231 and $\mathrm{A} \beta$ relationship was confined to $\mathrm{A} \beta$-positive individuals only $(P<0.001$, $\mathrm{r}=0.450)$. Plasma $\mathrm{p}$-tau231 was also highly correlated with CSF A $\beta_{1-42}(P<0.0001 ;$ Fig. $2 d)$. 
Fig. 1 Plasma p-tau231 concentration identifies individuals across the AD spectrum and neuropathologically confirmed AD cases. The box-and-whisker plots (left side) show plasma phospho-tau231 (p-tau231) concentrations across groups. For the box-and-whisker plots, the horizontal bar shows the median, and the upper and lower boundaries show the 25 th and 75 th percentiles, respectively. $P$ values indicate the results of the analysis of variance models with Tukey's post hoc multiple comparisons at $* * P<0.05$. The figure also displays the corresponding ROC curves in the four cohorts studied (right side) to separate individuals with $\mathrm{AD}$ dementia from the other groups. The AUC values of the ROC curves indicate the overall biomarker performance across groups, with 0.5 indicating no difference from chance and 1.0 a biomarker with specificity and sensitivity of $100 \%$. a, b In the discovery cohort $(n=38)$, plasma p-tau231 concentrations accurately discriminated $\mathrm{AD}$ from CU elderly $\mathrm{A} \beta$ - controls. $\mathbf{c}, \mathbf{d}$ In the TRIAD validation cohort ( $n=313)$, AD dementia individuals had higher plasma p-tau231 levels than all other groups. e, $\mathbf{f}$ In the primary care clinical cohort $(n=190)$, $\mathrm{AD}$ dementia individuals had higher plasma $\mathrm{p}$-tau231 than CU but not than MCI individuals. $\mathbf{g}, \mathbf{h}$ In the neuropathology cohort $(n=47)$ clinical cohort, $\mathrm{AD}$ individuals had higher plasma p-tau231 than non-AD dementias a Discovery cohort

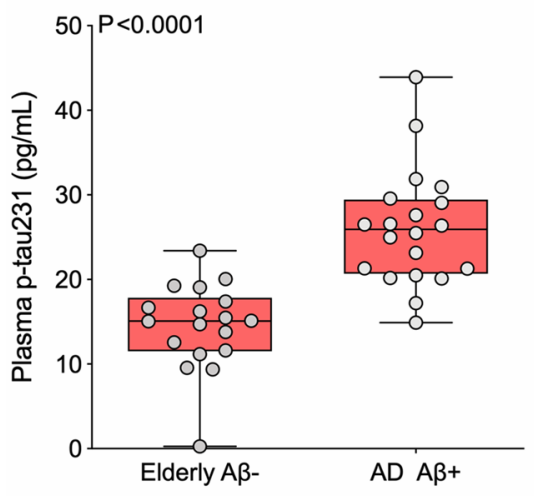

\section{c TRIAD cohort}

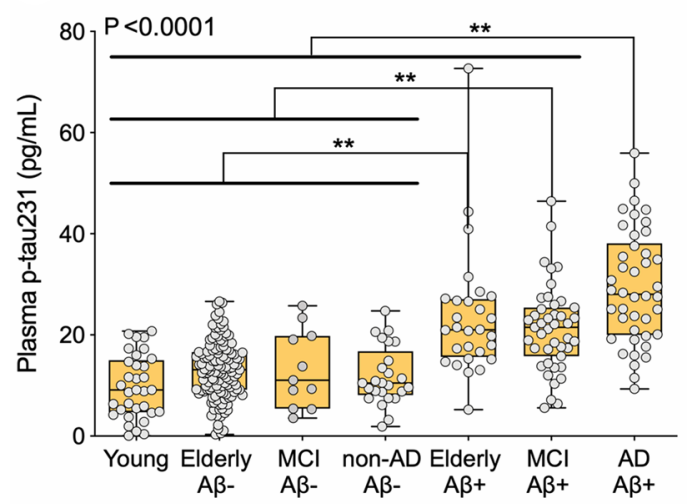

e Primary care

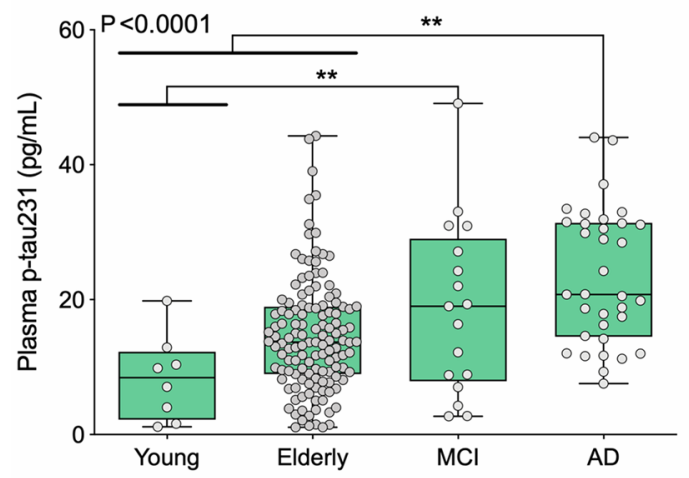

g Neuropathology cohort

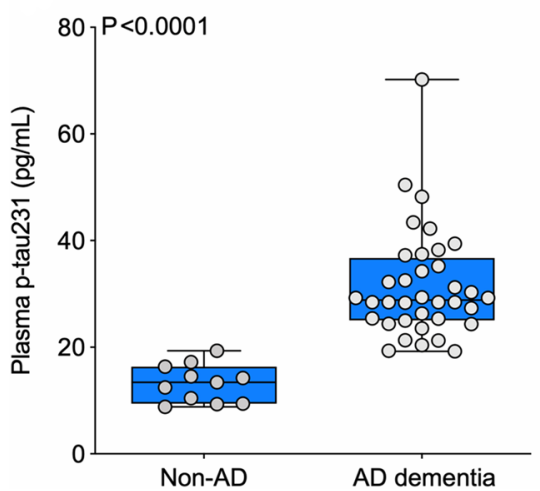

b Discovery cohort

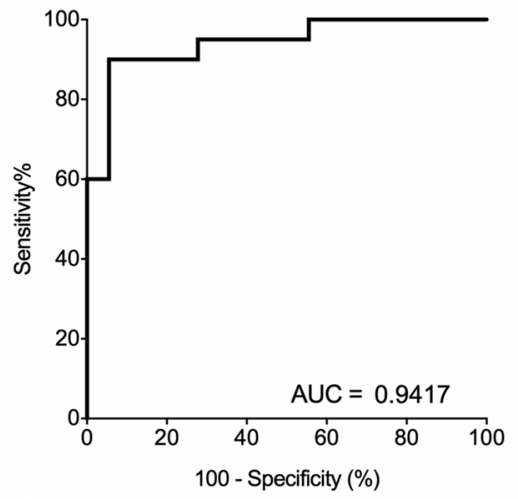

d TRIAD cohort

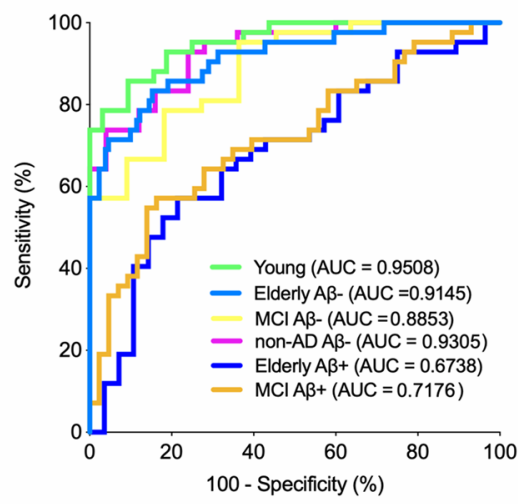

f Primary care

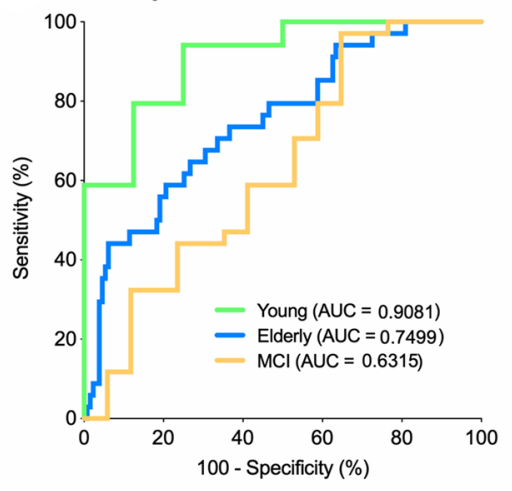

h Neuropathology cohort

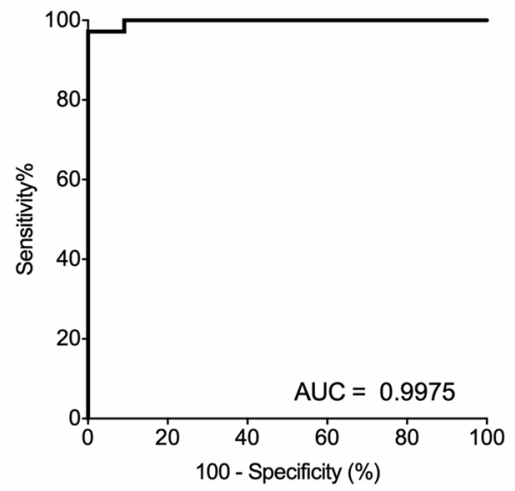


a Plasma p-tau231 correlates with tau PET

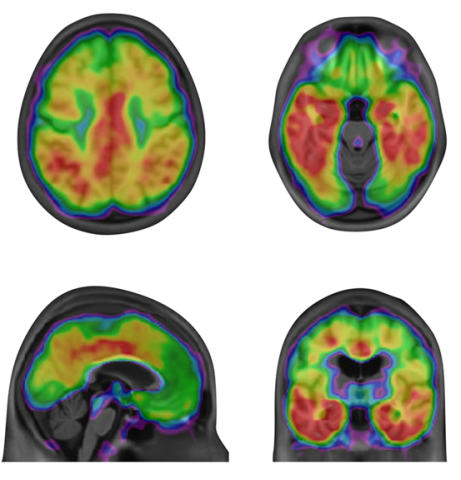

0 t-value 13

c Plasma p-tau231 correlates with $A \beta$ PET
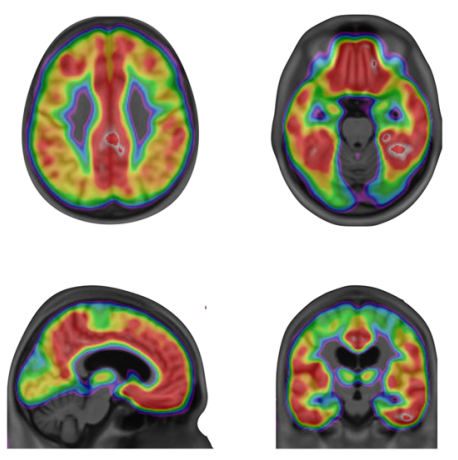

b Plasma and CSF p-tau231
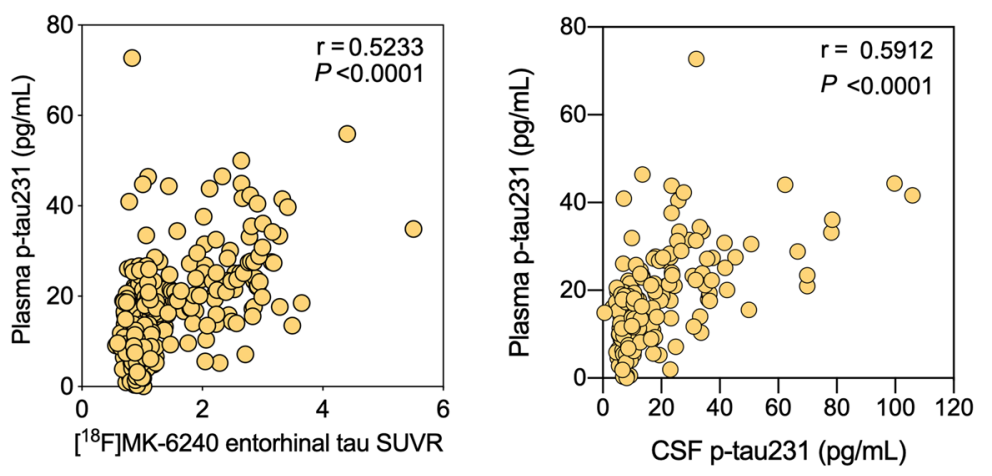

d Plasma p-tau231 and CSF A $\beta$

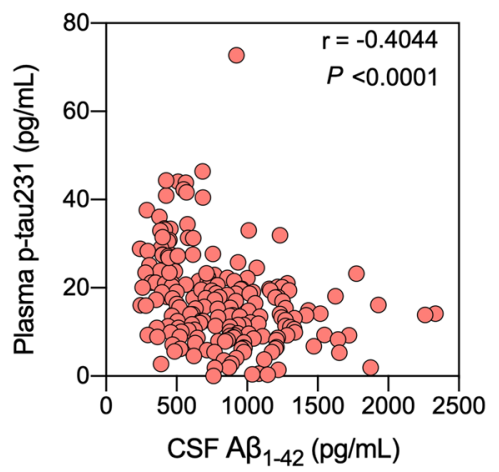

Fig. 2 Plasma p-tau231 concentration correlates with PET and CSF tau and $A \beta$. The brain maps show the results of linear regressions false discovery rate corrected for multiple comparisons at $P<0.05$, whereas the plots show the results of Spearman rank correlation between biomarkers. a Plasma p-tau231 concentrations were asso-

\section{Plasma p-tau231 as an early markers of AD pathology}

Plasma p-tau231 (AUC $=0.82$ (95\% CI 0.76-0.92)) differentiated CU A $\beta$-negative from CU A $\beta$-positive, and demonstrated a numerically higher AUC than plasma p-tau181 (supplementary Fig. 5, online resource). The inflection points of the weighted regression curves suggested that plasma p-tau231 demonstrates an earlier increase than plasma p-tau181 as a function of $\mathrm{A} \beta$ deposition load (Fig. 3) and that this was prior to the threshold of $A \beta$ positivity had been achieved ( $A \beta$ centiloid=22). Plasma p-tau231 showed abnormal levels from A $\beta$ PET quartiles 2-4, whereas plasma p-tau181 and CSF p-tau 217 became abnormal in quartile 4 and quartile 3, respectively (Fig. 4). Furthermore, plasma p-tau231 segregated individuals across early and late Braak stages of tau tangles accumulation (Fig. 5) including Braak I-II, which was not observed for plasma p-tau 181. ciated with $\left[{ }^{18} \mathrm{~F}\right] \mathrm{MK}-6240$ SUVR across the cortex with the highest association in the temporal and posterior cingulate cortices as well as with b CSF p-tau231 concentrations. c Plasma p-tau231 correlates with $\left[{ }^{18} \mathrm{~F}\right]$ AZD4694 SUVR across the cortex and with d CSF A $\beta_{1-42}$ concentrations

\section{Plasma p-tau231 predicts longitudinal AD progression}

A subsample of individuals in the TRIAD cohort $(n=126)$ had a 1-year follow-up structural MRI and cognitive assessment. Plasma p-tau231 predicted 1-year worsening in MMSE scores and hippocampal atrophy (Table 3).

\section{Discussion}

In this study, we report the first ultrasensitive immunoassay for the accurate quantification of phosphorylated tau at threonine 231 in plasma and investigated its potential in multiple scenarios across the AD continuum, primary care utility and against neuropathological confirmation. The findings show that plasma p-tau231, like plasma p-tau181, demonstrates high diagnostic accuracy in detecting AD in the dementia stage of the disease, which included neuropathologically 


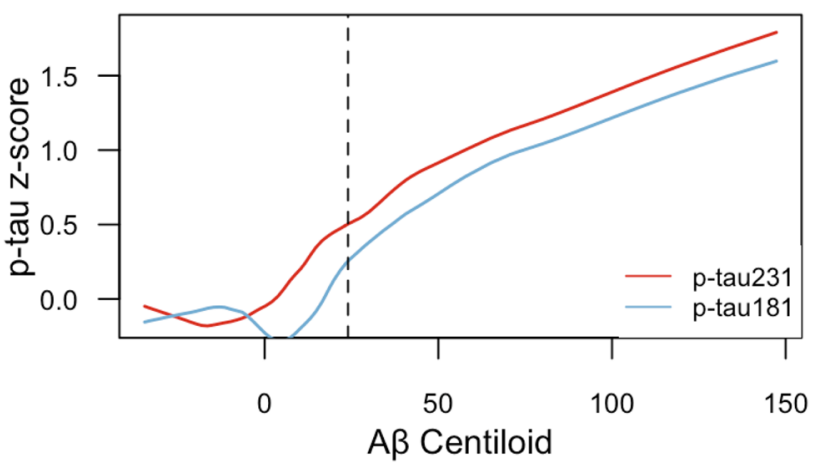

Fig. 3 Plasma p-tau231 increases earlier than plasma p-tau181 as a function of brain $\mathrm{A} \beta$ deposition. The lines represent weighted regression models showing the association between $z$-scored plasma p-tau231 and p-tau181 concentrations as a function of centiloid $\mathrm{A} \beta$ PET levels in $\mathrm{CU}$ and MCI individuals $(P<0.0001)$. The vertical dashed lines represent the inflection point of the plasma p-tau231 (red), plasma p-tau181 (blue), as well as the centiloid threshold of $\mathrm{A} \beta$ positivity (black; centiloid $=22$ ). The graph suggests that plasma p-tau231 raises before plasma p-tau181 with the increase of brain $A \beta$ levels

confirmed cases of $\mathrm{AD}$ and non-AD neurodegenerative diseases. Plasma p-tau231 also discriminated $A \beta$-positive $\mathrm{CU}$ and $\mathrm{MCI}$ cases from $\mathrm{A} \beta$-negative $\mathrm{CU}$ elderly with high accuracy and correlated strongly with $\mathrm{A} \beta$ and tau PET. The novel findings of plasma $p$-tau231 is that it was shown to (i) increase and correlate with A $\beta$ PET, prior to formal A $\beta$ PET positivity, (ii) significantly increases earlier than plasma p-tau181 in CU individuals, (iii) segregates A $\beta$ PET quartiles better than plasma p-tau 181 and CSF p-tau217 and iv) detects early tau deposition, which was not observed for plasma p-tau181.

The high specificity of CSF p-tau for AD is an established finding $[12,21,26,46]$. This conclusion is now an ever-increasing outcome in blood [10, 29, 34, 48] and makes plasma p-tau measures the main candidate for an AD-specific blood biomarker. To date, no report of plasma p-tau231 has been described in blood, despite it being a widely reported CSF biomarker $[2,11,14,15,18,20,30$, $33,53,58]$. The ultrasensitive Simoa assay presented in this study measures specific tau entities that include both the $\mathrm{N}$-terminal part of the molecule and a phosphorylated epitope including amino acid 231 (N-p-tau231, supplementary Fig. 1-2, online resource), as verified by mass spectrometry and immunoprecipitation experiments. Importantly, the strong correlation of plasma with CSF p-tau231 supports the notion that this assay specifically measures a brain-derived phospho-form of tau. Furthermore, as a proof-of-principle, our plasma p-tau231 assay has similar diagnostic performances as the previously described plasma p-tau181 assay [29] replicating the multitude of studies demonstrating that CSF p-tau231 and CSF p-tau181 have identical performances in the clinical stage of $\mathrm{AD}$ dementia. Specifically, plasma p-tau 231 had high diagnostic accuracy in distinguishing $\mathrm{AD}$ from non-AD dementias $(\mathrm{AUC}=0.93$ ), which included primary tauopathies (e.g., frontotemporal dementia, progressive supranuclear palsy and corticobasal degeneration) and this is most likely the main clinical application of plasma p-tau. This high discrimination between $\mathrm{AD}$ and non- $\mathrm{AD}$ was verified in patients with neuropathologically confirmed post-mortem tissue $(\mathrm{AUC}=0.99)$ and despite all individuals been given a clinical diagnosis of probable $\mathrm{AD}$ during life-confirming the potential clinical utility of plasma p-tau. Similarly, plasma p-tau231 could also discriminate $\mathrm{AD}$ from $\mathrm{MCI}$ cases without underlying $\mathrm{AD}$ pathology $(\mathrm{AUC}=0.89)$, which is also a potential challenge observed in clinical settings. Plasma p-tau231 also separated $A D$ from $A \beta$-negative $C U$ elderly $(A U C=0.92)$ and young adults $(A U C=0.95)$ with very high accuracy.
Plasma p-tau231

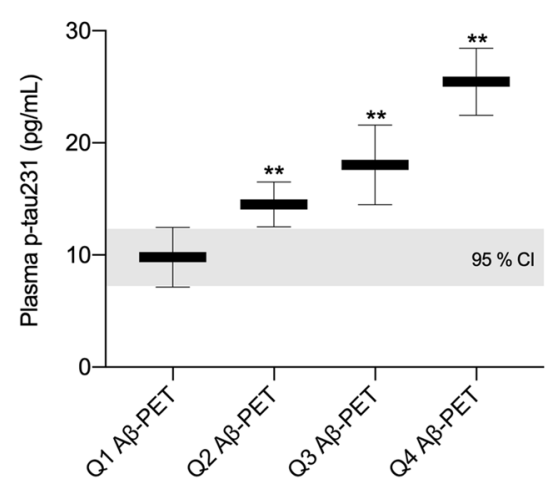

Plasma p-tau181

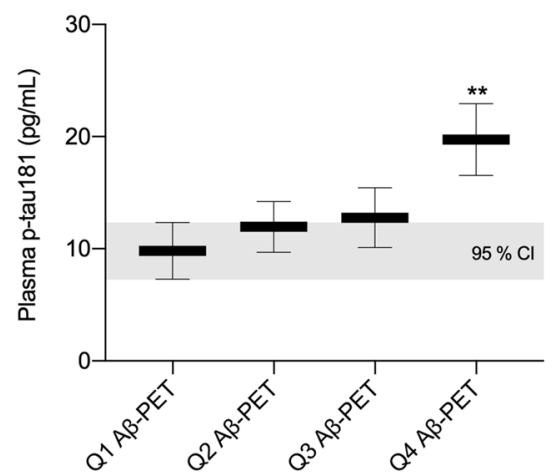

CSF p-tau217

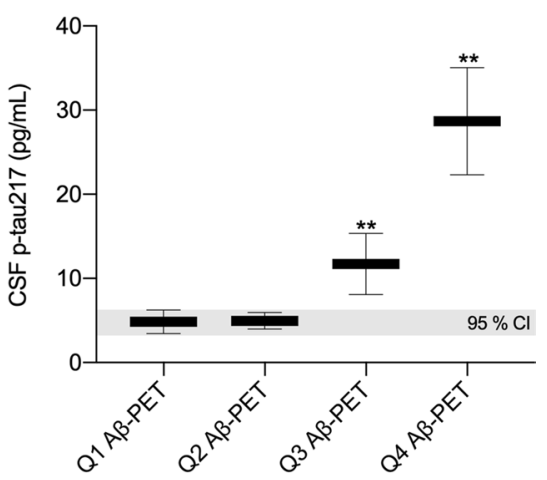

Fig. 4 Plasma p-tau231 increases earlier that plasma p-tau181 and CSF p-tau217 as a function of A $\beta$ PET quartiles. The horizontal grey bars shows the mean and 95\% confidence intervals (CI) of plasma p-tau231 and p-tau181 as well as CSF p-tau217 in elderly individuals (CU, MCI, AD) segregated A $\beta$ PET quartiles. Plasma p-tau231 showed abnormally increased levels from A $\beta$ PET quartile 2 to 4; plasma p-tau181 in quartile 4 , and CSF in quartiles 3-4 


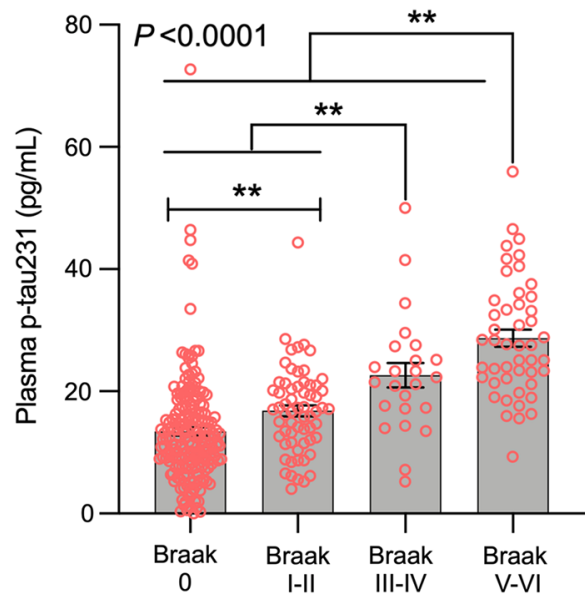

Fig. 5 Plasma p-tau231 concentration segregates individuals across the entire Braak stage spectrum. The bars show the mean and the standard error of the mean. $P$ values indicate the results of the analysis of variance models with Tukey's post hoc multiple comparisons at ** $P<0.05$. The figure shows that plasma $\mathrm{p}$-tau231 segregates indi-

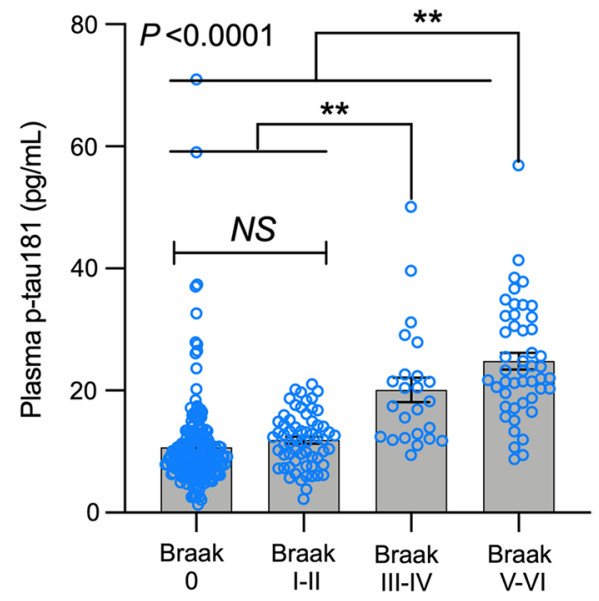

viduals across all Braak stage, whereas plasma p-tau181 did not differentiate individuals who were in Braak stage 0 from those in Braak stage I-II, suggesting plasma p-tau231 raises early in the AD pathophysiological phase

Table 3 Plasma p-tau231 concentration predicts longitudinal changes in hippocampus volume and cognitive decline

\begin{tabular}{|c|c|c|c|c|c|c|c|}
\hline \multicolumn{4}{|c|}{$\Delta \mathrm{HV} \sim \mathrm{P}$-tau $231+$ Age + Education $+\mathrm{Sex}+\mathrm{A} \beta$} & \multicolumn{4}{|c|}{$\Delta \mathrm{MMSE} \sim \mathrm{P}-\operatorname{tau} 231+$ Age + Education $+\mathrm{Sex}+\mathrm{A} \beta$} \\
\hline & Beta & Std. error & $p$ value & & Beta & Std. error & $p$ value \\
\hline P-tau231 & -0.0023 & 0.0011 & $0.0321 *$ & P-tau231 & -0.0424 & 0.0178 & $0.0196^{*}$ \\
\hline Age & -0.0003 & 0.0010 & 0.7340 & Age & 0.0310 & 0.0289 & 0.2866 \\
\hline Education & 0.0038 & 0.0028 & 0.1835 & Education & -0.0658 & 0.0502 & 0.1935 \\
\hline Sex & 0.0281 & 0.0208 & 0.1780 & Sex & -0.2403 & 0.3789 & 0.5275 \\
\hline $\mathrm{A} \beta$ & -0.0005 & 0.0034 & 0.8886 & $\mathrm{~A} \beta$ & -0.0244 & 0.0616 & 0.6934 \\
\hline \multicolumn{4}{|c|}{$\overline{\Delta H V \sim \text { P-tau181 + Age + Education }+ \text { Sex }+ \text { A } \beta}$} & \multicolumn{4}{|c|}{$\Delta \mathrm{MMSE} \sim$ P-tau181 + Age + Education $+\mathrm{Sex}+\mathrm{A} \beta$} \\
\hline & Beta & Std. error & $p$ value & & Beta & Std. error & $p$ value \\
\hline P-tau181 & -0.0025 & 0.0012 & $0.0452 *$ & $\mathrm{P}-\operatorname{tau} 181$ & -0.0751 & 0.0216 & $0.0008^{*}$ \\
\hline Age & -0.0005 & 0.0010 & 0.6335 & Age & 0.0259 & 0.0278 & 0.3542 \\
\hline Education & 0.0036 & 0.0029 & 0.2151 & Education & -0.0657 & 0.0487 & 0.1808 \\
\hline Sex & 0.0307 & 0.0207 & 0.1398 & Sex & -0.1762 & 0.3643 & 0.6298 \\
\hline $\mathrm{A} \beta$ & -0.0002 & 0.0034 & 0.9618 & $\mathrm{~A} \beta$ & -0.0106 & 0.0594 & 0.8588 \\
\hline
\end{tabular}

The table show the results of beta values, standard error, and $\mathrm{p}$ values coefficients of linear regression models. Both plasma $\mathrm{p}-$ tau231 and $\mathrm{p}-$ tau181 precicted with 1- year decrease in hippocampal volume (HV) (neurodegeneration) and 1- year worsening in MMSE scores (cognitive decline) in a subset of TRIAD individuals $(n=126)$, accounting for years of age, years of formal education, sex (female $=1)$, and plasma A $\beta$ levels. For changes $(\Delta)$ in MMSE score and HV, lower scores represent a cognitive decline and decrease in brain volume, respectively

However, it demonstrated, much like the CSF comparisons that have preceded this study, that plasma p-tau231 was not significantly superior nor inferior to plasma p-tau181 in the aforementioned analysis. One exception to this being plasma p-tau231 being able to distinguish CU A $\beta$-positive participants from $A \beta$-negative $\mathrm{MCI}$ cases with higher accuracy and significantly superior to plasma p-tau181 (supplementary table 7 , online resource).
As the performance of plasma p-tau231 was shown to be equivalent to plasma p-tau181 in several domains (e.g., distinguishing $\mathrm{AD}$ and non- $\mathrm{AD}$ and identifying $\mathrm{A} \beta$ positivity), and combining these biomarkers did not show significant improvement in their performance in the cases tested in this study — what does a measure of plasma p-tau231 offer compared to other plasma p-tau biomarkers that have been previously described? Recently, we performed two independent studies comparing CSF p-tau biomarkers (p-tau181, 
p-tau217, p-tau231) in the AD continuum. Suarez-Calvet et al. [58] demonstrated, in a CU preclinical cohort, that CSF p-tau231 was increased more prominently with emerging pathology, whether $A \beta$ pathophysiology was measured in CSF or using PET. In support of this, CSF p-tau231 had a markedly stronger relationship with increasing $A \beta$ in individuals prior to the threshold of $A \beta$ positivity [3]. These associations were localized to focal $A \beta$ retention in brain regions corresponding to regions involved in the default mode network, a previously reported site of early $\mathrm{A} \beta$ pathology accumulation [49]. Neuropathological findings also highlight tau phosphorylation at threonine 231 to be a key marker of early disease as it is a predominate feature of preneurofibrillary tangle pathology [19] and one of the earliest events in the cascade of phosphorylation that modulates tubulin assembly $[1,55]$. Together, these recent CSF findings and neuropathological evidence paired with the successful replication of p-tau biomarkers from CSF-to-blood, we hypothesized that plasma p-tau231 would indeed mirror a very early increase in the $\mathrm{AD}$ continuum. We observed a numerically higher AUC of plasma p-tau231 as compared to $p$-tau 181 in the prediction of $A \beta$ positivity in $C U$ individuals, which mirrors CSF findings [58]. However, no significant differences between plasma p-tau231 and p-tau181 were found in this analysis, likely attributed to the finding that soluble p-tau 231 is already increasing in relation to $A \beta$ pathology prior to $A \beta$ PET positivity. We demonstrated this by showing that the inflection point of p-tau231, and consequent increase, occurs considerably earlier than plasma p-tau181 as a function of brain $A \beta$ deposition in dementia-free participants-this change was substantially earlier than the threshold of centiloid A $\beta$ PET positivity. Moreover, plasma $p$-tau231 shows a progressive stepwise increase with $A \beta$ levels in $A \beta$-negative individuals which was not observed for plasma p-tau181 and even CSF p-tau217.

The early and pronounced increase of plasma p-tau231, as shown in this study, has obvious implications for therapeutic trials, which are increasingly seeking to recruit individuals in the earliest stages of $\mathrm{AD}$ pathophysiology. $\mathrm{A} \beta$ deposits are a relatively late consequence of $A \beta$ aggregation in $\mathrm{AD}$ - thus the preclinical phase of $\mathrm{AD}(\mathrm{A} \beta$ deposition is present with no clinical symptoms) is the desired population target of many antibody-mediated clearances of $\mathrm{A} \beta$. Plasma biomarkers have been proposed to act as a prescreening tool to detect $A \beta$-positive individuals. In our recent analysis on the ADNI cohort, we described the substantial cost-savings that a robust plasma biomarker (e.g., plasma p-tau181) could have if introduced into the recruiting process of $\mathrm{CU}$ individuals with $\mathrm{A} \beta$ pathology [27]. The present study suggests that plasma p-tau 231 may be superior at this task $\left(\mathrm{AUC}_{\mathrm{p} \text {-tau231 }}=0.83\right.$ versus $\mathrm{AUC}=0.77_{\mathrm{p} \text {-tau } 181}$ in this study or $\mathrm{AUC}_{\mathrm{p} \text {-tau181 }}=0.70$ as reported by Karikari et al. [27]). However, recent in vitro evidence demonstrates that pre-clinical AD may appear as a consequence of a much earlier pathogenic events, prior any in vivo detection of $A \beta$ aggregation, and by reducing the concentration of $A \beta$ during a "pre-amyloid" phase halts $\mathrm{A} \beta$ plaque formation and $\mathrm{AD}$ onset [63]. Therefore, the pre-clinical phase of $\mathrm{AD}$ maybe a too advanced state in the disease course for the therapeutic agent to optimally target the most pathogenic phase of the disease. We have shown that plasma p-tau231 can stage $A \beta$-negative individuals based on their proximity to the threshold for $\mathrm{A} \beta$-positivity. Therefore, a critical use of plasma p-tau 231 would be a pre-screen to identify participants who have higher levels of $A \beta$ burden but who have not reached the threshold of positivity by PET (quartile 2 or 3 , in our study)—namely, the "pre-amyloid" phase. Our analysis suggests that only plasma p-tau 231 can identify both quartile 2 and quartile 3 from quartile 1 , whereas plasma p-tau 181 cannot distinguish between quartiles 1-3 and CSF p-tau217 (inferring on plasma p-tau217) can identify quartiles 3 and 4 but not quartile 2 . This distinctly shows the early increases of plasma p-tau231 and the potential to rapidly highlight a risk group, an intermediate between $A \beta$-negative and $\mathrm{A} \beta$-positive, which would be attractive and more beneficial group to target with anti-A $\beta$ therapeutics. Further, a negative plasma p-tau231 test (e.g., quartile 1, Fig. 4) not only indicates no clinical $\mathrm{AD}$ (and $\mathrm{A} \beta$-negativity) but also suggests no emerging pathology is present-thus, would satisfy as definitive confirmation of controls for a trial design, interventions and research cohorts. At the very least, individuals highlighted as quartile 3 by plasma p-tau 231 would not be adequate as definitive $A \beta$-negative controls.

In light of the marginal clinical benefit of the clinical trials with $A \beta$-targeting drugs [61] and findings that tau pathology is more related to the clinical presentation of $\mathrm{AD}$ - tau therapies are emerging as possible frontrunners in the search for an effective treatment for AD [24]. Nevertheless, common issues with anti-A $\beta$ trial recruitment are likely to occur in the anti-tau trials as well, e.g., methods to identify underlying pathology are too costly and invasive. Plasma p-tau231 strongly associated with tau PET demonstrating its relationship with insoluble tau deposits. Plasma p-tau 231 was associated with $\left[{ }^{18} \mathrm{~F}\right] \mathrm{MK}-6240$ retention in all tested regions but was strongest in the entorhinal cortex (Braak I-II) which is one of the earliest regions affected in $\mathrm{AD}$ and a site of early tau deposition. When employing the Braak staging system by tau PET, plasma p-tau231 segregated individuals across early and late Braak stages of tau tangle accumulation in a stepwise manner. This was also observed in the neuropathology cohort, despite being a small number of subjects in the Braak I-II group. Importantly, in the validation cohort, a significant increase of plasma p-tau231 was observed in Braak I-II as compared to Braak 0 (tau PET-negative), which was not observed for plasma p-tau181. This earlier 
increase has previously been described as an important feature of CSF p-tau231 [16], which is now replicated in blood. The non-significant difference observed for plasma p-tau 181 at early Braak stages (Braak 0 versus Braak I-II) has been verified by other groups [25, 60]. This demonstrates that plasma p-tau231 could act as an early marker of aberrant tau phosphorylation but also insoluble tau deposition, which can be utilised in future tau interventional studies. The biomarker could also be used in genetic and epidemiological studies to find novel risk and resilience factors specific for AD-type neuropathology, and validate whether those associated with dementia are specifically related to $\mathrm{AD}$.

Tau in CSF and plasma is predominantly $\mathrm{N}$-terminal to mid-region truncated fragments [7, 54]. Furthermore, biochemical and mass spectrometry methods show that the phosphorylation profile of soluble tau in AD brain was highly correlated to that in AD CSF [22]. Thus, future longitudinal studies in specific stages of AD may reveal a specific role for specific phospho-tau forms in the evolution of that stage e.g., p-tau231 and p-tau217 being early markers linking $A \beta$ and tau pathology before tau PET becomes detectable, while in the AD dementia stage, decreases in tau phosphorylated at serine 202 and p-tau 231 may reflect the 'transition' of soluble tau into insoluble tau in $\mathrm{AD}$ brain [22].

A limitation of this study is that we cannot directly compare plasma p-tau231 with plasma p-tau217. However, using CSF p-tau217 as a substitute for plasma p-tau217 (which is very likely a stronger predictor of disease pathophysiology) we are confident in our conclusions. Although our findings show that plasma p-tau 231 assay can identify $\mathrm{AD}$ in a primary care setting, the primary care cohort had no confirmatory biomarkers, of individuals with MCI and identification of preclinical AD in CU adults. Given the early increases in p-tau231, these assessments would have reduced the overlap in plasma p-tau231 concentrations between $\mathrm{CU}, \mathrm{MCI}$ and AD. Furthermore, the use of cross-sectional A $\beta$ PET Centiloids as a proxy of time in the disease was employed and it is not guaranteed that a greater A $\beta$ PET SUVR is indicative of more advanced disease state. Lastly, to definitively and fully describe the ordinal sequence of plasma p-tau biomarkers, from preclinical to symptomatic phases of the disease, large-scale longitudinal studies with multiple time points are required.

To conclude, our novel plasma p-tau231 assay is the first description of this tau phosphorylation site in the blood which identifies AD pathology, at the dementia stage, to the same degree as plasma p-tau181. However, plasma p-tau231 has additional advantages; (1) begins to increase with subtle $A \beta$ deposition, prior to $A \beta$ PET threshold has been attained, and noticeably before p-tau 181 and segregates A $\beta$ PET better than p-tau217; (2) increases in p-tau231 with early NFT deposition in the entorhinal cortex (Braak 0 versus Braak I-II) which not observed for $\mathrm{p}$-tau181. Thus, plasma p-tau231 demonstrates excellent clinical utility as a rapid screening test for $\mathrm{AD}$ but may serve as a superior staging biomarker of emerging AD pathology allowing clinical trials to targeting vulnerable populations under the threshold of $A \beta$ positivity and early tau deposition.

Supplementary Information The online version contains supplementary material available at https://doi.org/10.1007/s00401-021-02275-6.

Acknowledgments The authors thank all participants of the study and staff at the University of Gothenburg, Sahlgrenska University Hospital, McGill University Research Centre for Studies, and Montreal Neurological Institute who supported this project. We thank Cerveau Technologies for MK-6240, and to GE Healthcare for providing the precursor of flutemetamol. We also thank Kimberley Mauroo for her assistance in the ADx205 epitope mapping. Tissue samples were supplied by The London Neurodegenerative Diseases Brain Bank, which receives funding from the UK Medical Research Council and as part of the Brains for Dementia Research programme, jointly funded by Alzheimer's Research UK and the Alzheimer's Society.

Author contributions NJA, TAP, TKK, HZ, PR-N, and KB conceived the study. NJA and TKK developed and validated the blood assay for tau phosphorylated at threonine 231 with support from JLR, GB, EV, $\mathrm{HZ}$, and KB. NJA and TAP performed the statistical analysis. TAP, ALB, and PR-N designed and implemented MRI and PET acquisition protocols, and performed image processing and quality control. SG, $\mathrm{HZ}, \mathrm{PR}-\mathrm{N}$ and KB recruited participants and collected clinical data. NJA, TAP, TKK, ALB, SG, HZ, PR-N, and KB interpreted the data. NJA, TAP and KB drafted the initial manuscript. All authors contributed to the revision and editing of the manuscript.

Funding Open Access funding provided by University of Gothenburg. NJA is supported by the Wallenberg Centre for Molecular and Translational Medicine, Swedish Alzheimer Foundation (Alzheimerfonden), Swedish Brain Foundation (Hjärnfonden), Swedish Dementia Foundation (Demensfonden) and Gamla Tjänarinnor. TAP was supported by the Alzheimer Association (AACSF-20-648075). TKK is supported by the BrightFocus Foundation (\#A2020812F), the Swedish Alzheimer Foundation (Alzheimerfonden; \#AF-930627), the Swedish Brain Foundation (Hjärnfonden; \#FO2020-0240), the Swedish Parkinson Foundation (Parkinsonfonden; \#1252/20), the Swedish Dementia Foundation (Demensförbundet), Gamla Tjänarinnor Foundation, the Aina (Ann) Wallströms and Mary-Ann Sjöbloms Foundation, the Agneta Prytz-Folkes \& Gösta Folkes Foundation (\#2020-00124), the Gun and Bertil Stohnes Foundation, and the Anna Lisa and Brother Björnsson's Foundation. HZ is a Wallenberg Scholar supported by grants from the Swedish Research Council (\#2018-02532), the European Research Council (\#681712), Swedish State Support for Clinical Research (\#ALFGBG-720931), the Alzheimer Drug Discovery Foundation (ADDF), USA (\#201809-2016862), the European Union's Horizon 2020 research and innovation programme under the Marie Skłodowska-Curie grant agreement No 860197 (MIRIADE), and the UK Dementia Research Institute at UCL. TRIAD is supported by the Canadian Institutes of Health Research (CIHR) [MOP-11-51-31; RFN 152985, 159815, 162303], Canadian Consortium of Neurodegeneration and Aging (CCNA; MOP-11-51-31 -team 1), Weston Brain Institute, Brain Canada Foundation (CFI Project 34874; 33397), the Fonds de Recherche du Québec-Santé (FRQS; Chercheur Boursier, 2020-VICO-279314). TAP, P.R-N and SG are members of the CIHRCCNA Canadian Consortium of Neurodegeneration in Aging. KB 
was supported by the Alzheimer Drug Discovery Foundation (ADDF; \#RDAPB-201809-2016615), the Swedish Research Council (\#201700915), the Swedish Alzheimer Foundation (\#AF-742881), Hjärnfonden, Sweden (\#FO2017-0243), and a grant (\#ALFGBG-715986) from the Swedish state under the agreement between the Swedish government and the County Councils, the ALF-agreement.

\section{Compliance with ethical standards}

Conflict of interest HZ has served at scientific advisory boards for Denali, Roche Diagnostics, Wave, Samumed, Siemens Healthineers, Pinteon Therapeutics and $\operatorname{CogRx}$, and has given lectures in symposia sponsored by Fujirebio, Alzecure and Biogen. KB has served as a consultant, at advisory boards, or at data monitoring committees for Abcam, Axon, Biogen, JOMDD/Shimadzu. Julius Clinical, Lilly, $\mathrm{MagQu}$, Novartis, Roche Diagnostics, and Siemens Healthineers, and is a co-founder of Brain Biomarker Solutions in Gothenburg AB (BBS), which is a part of the GU Ventures Incubator Program. EVM is a co-founder of ADx NeuroSciences. The other authors declare no competing interest.

Open Access This article is licensed under a Creative Commons Attribution 4.0 International License, which permits use, sharing, adaptation, distribution and reproduction in any medium or format, as long as you give appropriate credit to the original author(s) and the source, provide a link to the Creative Commons licence, and indicate if changes were made. The images or other third party material in this article are included in the article's Creative Commons licence, unless indicated otherwise in a credit line to the material. If material is not included in the article's Creative Commons licence and your intended use is not permitted by statutory regulation or exceeds the permitted use, you will need to obtain permission directly from the copyright holder. To view a copy of this licence, visit http://creativecommons.org/licenses/by/4.0/.

\section{References}

1. Amniai L, Barbier P, Sillen A, Wieruszeski JM, Peyrot V, Lippens G, Landrieu I (2009) Alzheimer disease specific phosphoepitopes of Tau interfere with assembly of tubulin but not binding to microtubules. FASEB J 23:1146-1152. https://doi.org/10.1096/ fj. $08-121590$

2. Arai $\mathrm{H}$, Ishiguro $\mathrm{K}$, Ohno $\mathrm{H}$, Moriyama $\mathrm{M}$, Itoh N, Okamura N, Matsui T, Morikawa Y, Horikawa E, Kohno H et al (2000) CSF phosphorylated tau protein and mild cognitive impairment: a prospective study. Exp Neurol 166:201-203. https://doi.org/10.1006/ exnr.2000.7501

3. Ashton NJ, Benedet AL, Pascoal TA, Karikari TK, Lantero-Rodriguez J, Mathotaarachchi S, Therriault J, Savard M, Chamoun M, Stoops E et al (2021) Cerebrospinal fluid p-tau231 as an early indicator of emerging pathology in Alzheimer's disease. Research Square. https://doi.org/10.21203/rs.3.rs-155736/v1 (PREPRINT (Version 1))

4. Ashton NJ, Janelidze S, Al-Khleifat A, Leuzy A, Van der Ende EL, Karikari TK, Benedet AL, Pascoal TA, Lleó A, Parnetti L et al (2020) Diagnostic value of plasma neurofilament light: a multicentre validation study. Research Square. https://doi.org/10.21203 /rs.3.rs-63386/v1 (PREPRINT (Version 1))

5. Ashton NJ, Leuzy A, Lim YM, Troakes C, Hortobagyi T, Hoglund K, Aarsland D, Lovestone S, Scholl M, Blennow K et al (2019) Increased plasma neurofilament light chain concentration correlates with severity of post-mortem neurofibrillary tangle pathology and neurodegeneration. Acta Neuropathol Commun 7:5. https://doi.org/10.1186/s40478-018-0649-3

6. Barthelemy NR, Bateman RJ, Hirtz C, Marin P, Becher F, Sato C, Gabelle A, Lehmann S (2020) Cerebrospinal fluid phospho-tau T217 outperforms T181 as a biomarker for the differential diagnosis of Alzheimer's disease and PET amyloid-positive patient identification. Alzheimers Res Ther 12:26. https://doi.org/10.1186/ s13195-020-00596-4

7. Barthelemy NR, Horie K, Sato C, Bateman RJ (2020) Blood plasma phosphorylated-tau isoforms track CNS change in Alzheimer's disease. J Exp Med. https://doi.org/10.1084/jem.20200 861

8. Bateman RJ, Xiong C, Benzinger TL, Fagan AM, Goate A, Fox NC, Marcus DS, Cairns NJ, Xie X, Blazey TM et al (2012) Clinical and biomarker changes in dominantly inherited Alzheimer's disease. N Engl J Med 367:795-804. https://doi.org/10.1056/ NEJMoa1202753

9. Benedet AL, Leuzy A, Pascoal TA, Ashton NJ, Mathotaarachchi S, Savard M, Therriault J, Kang MS, Chamoun M, Scholl M et al (2020) Stage-specific links between plasma neurofilament light and imaging biomarkers of Alzheimer's disease. Brain. https:// doi.org/10.1093/brain/awaa342

10. Benussi A, Karikari TK, Ashton N, Gazzina S, Premi E, Benussi L, Ghidoni R, Rodriguez JL, Emersic A, Simren J et al (2020) Diagnostic and prognostic value of serum NfL and p-Tau181 in frontotemporal lobar degeneration. J Neurol Neurosurg Psychiatry 91:960-967. https://doi.org/10.1136/jnnp-2020-323487

11. Blennow K, Vanmechelen E, Hampel H (2001) CSF total tau, Abeta 42 and phosphorylated tau protein as biomarkers for Alzheimer's disease. Mol Neurobiol 24:87-97. https://doi.org/10.1385/ MN:24:1-3:087

12. Blennow K, Zetterberg H (2018) Biomarkers for Alzheimer's disease: current status and prospects for the future. J Intern Med 284:643-663. https://doi.org/10.1111/joim. 12816

13. Braak H, Alafuzoff I, Arzberger T, Kretzschmar H, Del Tredici K (2006) Staging of Alzheimer disease-associated neurofibrillary pathology using paraffin sections and immunocytochemistry. Acta Neuropathol 112:389-404. https://doi.org/10.1007/s0040 1-006-0127-z

14. Brys M, Pirraglia E, Rich K, Rolstad S, Mosconi L, Switalski R, Glodzik-Sobanska L, De Santi S, Zinkowski R, Mehta P et al (2009) Prediction and longitudinal study of CSF biomarkers in mild cognitive impairment. Neurobiol Aging 30:682-690. https ://doi.org/10.1016/j.neurobiolaging.2007.08.010

15. Buerger K, Teipel SJ, Zinkowski R, Blennow K, Arai H, Engel R, Hofmann-Kiefer K, McCulloch C, Ptok U, Heun R et al (2002) CSF tau protein phosphorylated at threonine 231 correlates with cognitive decline in MCI subjects. Neurology 59:627-629. https ://doi.org/10.1212/wnl.59.4.627

16. Castellani RJ, Perry G (2019) Tau biology, tauopathy, traumatic brain injury, and diagnostic challenges. J Alzheimers Dis 67:447467. https://doi.org/10.3233/JAD-180721

17. Cleveland WS (1979) Robust locally weighted regression and smoothing scatterplots. Journal of the American statistical association 74:829-836

18. de Leon MJ, Segal S, Tarshish CY, DeSanti S, Zinkowski R, Mehta PD, Convit A, Caraos C, Rusinek H, Tsui W et al (2002) Longitudinal cerebrospinal fluid tau load increases in mild cognitive impairment. Neurosci Lett 333:183-186. https://doi. org/10.1016/s0304-3940(02)01038-8

19. Ercan-Herbst E, Ehrig J, Schondorf DC, Behrendt A, Klaus B, Gomez Ramos B, Prat Oriol N, Weber C, Ehrnhoefer DE (2019) A post-translational modification signature defines changes in soluble tau correlating with oligomerization in early stage Alzheimer's disease brain. Acta Neuropathol Commun 7:192. https ://doi.org/10.1186/s40478-019-0823-2 
20. Hampel H, Burger K, Pruessner JC, Zinkowski R, DeBernardis J, Kerkman D, Leinsinger G, Evans AC, Davies P, Moller HJ et al (2005) Correlation of cerebrospinal fluid levels of tau protein phosphorylated at threonine 231 with rates of hippocampal atrophy in Alzheimer disease. Arch Neurol 62:770-773. https:// doi.org/10.1001/archneur.62.5.770

21. Hanes J, Kovac A, Kvartsberg H, Kontsekova E, Fialova L, Katina S, Kovacech B, Stevens E, Hort J, Vyhnalek M et al (2020) Evaluation of a novel immunoassay to detect p-Tau Thr217 in the CSF to distinguish Alzheimer disease from other dementias. Neurology. https://doi.org/10.1212/WNL.0000000000010814

22. Horie K, Barthelemy NR, Mallipeddi N, Li Y, Franklin EE, Perrin RJ, Bateman RJ, Sato C (2020) Regional correlation of biochemical measures of amyloid and tau phosphorylation in the brain. Acta Neuropathol Commun 8:149. https://doi.org/10.1186/s4047 8-020-01019-Z

23. Jack CR Jr, Bennett DA, Blennow K, Carrillo MC, Dunn B, Haeberlein SB, Holtzman DM, Jagust W, Jessen F, Karlawish J et al (2018) NIA-AA Research Framework: Toward a biological definition of Alzheimer's disease. Alzheimers Dement 14:535-562. https://doi.org/10.1016/j.jalz.2018.02.018

24. Jadhav S, Avila J, Scholl M, Kovacs GG, Kovari E, Skrabana R, Evans LD, Kontsekova E, Malawska B, de Silva R et al (2019) A walk through tau therapeutic strategies. Acta Neuropathol Commun 7:22. https://doi.org/10.1186/s40478-019-0664-z

25. Janelidze S, Mattsson N, Palmqvist S, Smith R, Beach TG, Serrano GE, Chai X, Proctor NK, Eichenlaub U, Zetterberg $\mathrm{H}$ et al (2020) Plasma P-tau181 in Alzheimer's disease: relationship to other biomarkers, differential diagnosis, neuropathology and longitudinal progression to Alzheimer's dementia. Nat Med 26:379386. https://doi.org/10.1038/s41591-020-0755-1

26. Janelidze S, Stomrud E, Smith R, Palmqvist S, Mattsson N, Airey DC, Proctor NK, Chai X, Shcherbinin S, Sims JR et al (2020) Cerebrospinal fluid p-tau217 performs better than p-tau181 as a biomarker of Alzheimer's disease. Nat Commun 11:1683. https://doi.org/10.1038/s41467-020-15436-0

27. Karikari TK, Benedet AL, Ashton NJ, Lantero Rodriguez J, Snellman A, Suarez-Calvet M, Saha-Chaudhuri P, Lussier F, Kvartsberg H, Rial AM et al (2020) Diagnostic performance and prediction of clinical progression of plasma phospho-tau181 in the Alzheimer's Disease Neuroimaging Initiative. Mol Psychiatry. https://doi.org/10.1038/s41380-020-00923-z

28. Karikari TK, Emersic A, Vrillon A, Lantero-Rodriguez J, Ashton NJ, Kramberger MG, Dumurgier J, Hourregue C, Cucnik S, Brinkmalm G et al (2020) Head-to-head comparison of clinical performance of CSF phospho-tau T181 and T217 biomarkers for Alzheimer's disease diagnosis. Alzheimers Dement. https://doi.org/10.1002/alz.12236

29. Karikari TK, Pascoal TA, Ashton NJ, Janelidze S, Benedet AL, Rodriguez JL, Chamoun M, Savard M, Kang MS, Therriault J et al (2020) Blood phosphorylated tau 181 as a biomarker for Alzheimer's disease: a diagnostic performance and prediction modelling study using data from four prospective cohorts. Lancet Neurol 19:422-433. https://doi.org/10.1016/S1474 $-4422(20) 30071-5$

30. Kidemet-Piskac S, Babic Leko M, Blazekovic A, Langer Horvat L, Klepac N, Sonicki Z, Kolenc D, Hof PR, Boban M, Mimica $\mathrm{N}$ et al (2018) Evaluation of cerebrospinal fluid phosphorylated tau231 as a biomarker in the differential diagnosis of Alzheimer's disease and vascular dementia. CNS Neurosci Ther 24:734-740. https://doi.org/10.1111/cns.12814

31. Klein A, Tourville J (2012) 101 labeled brain images and a consistent human cortical labeling protocol. Front Neurosci 6:171. https://doi.org/10.3389/fnins.2012.00171

32. Klunk WE, Koeppe RA, Price JC, Benzinger TL, Devous MD Sr, Jagust WJ, Johnson KA, Mathis CA, Minhas D, Pontecorvo MJ et al (2015) The Centiloid Project: standardizing quantitative amyloid plaque estimation by PET. Alzheimers Dement 11(1-15):e1114. https://doi.org/10.1016/j.jalz.2014.07.003

33. Kohnken R, Buerger K, Zinkowski R, Miller C, Kerkman D, DeBernardis J, Shen J, Moller HJ, Davies P, Hampel H (2000) Detection of tau phosphorylated at threonine 231 in cerebrospinal fluid of Alzheimer's disease patients. Neurosci Lett 287:187-190. https://doi.org/10.1016/s0304-3940(00)01178-2

34. Lantero Rodriguez J, Karikari TK, Suarez-Calvet M, Troakes C, King A, Emersic A, Aarsland D, Hye A, Zetterberg H, Blennow K et al (2020) Plasma p-tau181 accurately predicts Alzheimer's disease pathology at least 8 years prior to post-mortem and improves the clinical characterisation of cognitive decline. Acta Neuropathol 140:267-278. https://doi.org/10.1007/s00401-02002195-X

35. Leuzy A, Smith R, Ossenkoppele R, Santillo A, Borroni E, Klein G, Ohlsson T, Jogi J, Palmqvist S, Mattsson-Carlgren N et al (2020) Diagnostic Performance of RO948 F 18 Tau positron emission tomography in the differentiation of Alzheimer disease from other neurodegenerative disorders. JAMA Neurol 77:955-965. https://doi.org/10.1001/jamaneurol.2020.0989

36. Mathotaarachchi S, Wang S, Shin M, Pascoal TA, Benedet AL, Kang MS, Beaudry T, Fonov VS, Gauthier S, Labbe A et al (2016) VoxelStats: a matlab package for multi-modal voxel-wise brain image analysis. Front Neuroinform 10:20. https://doi.org/10.3389/ fninf.2016.00020

37. Mattsson N, Andreasson U, Zetterberg H, Blennow K, Alzheimer's Disease Neuroimaging I (2017) Association of plasma neurofilament light with neurodegeneration in patients with Alzheimer disease. JAMA Neurol 74:557-566. https://doi.org/10.1001/ jamaneurol.2016.6117

38. Mattsson-Carlgren N, Janelidze S, Palmqvist S, Cullen N, Svenningsson AL, Strandberg O, Mengel D, Walsh DM, Stomrud E, Dage JL et al (2020) Longitudinal plasma p-tau217 is increased in early stages of Alzheimer's disease. Brain. https://doi.org/10.1093/ brain/awaa286

39. McKhann G, Drachman D, Folstein M, Katzman R, Price D, Stadlan EM (1984) Clinical diagnosis of Alzheimer's disease: report of the NINCDS-ADRDA Work Group under the auspices of Department of Health and Human Services Task Force on Alzheimer's Disease. Neurology 34:939-944. https://doi.org/10.1212/ wnl.34.7.939

40. McKhann GM, Knopman DS, Chertkow H, Hyman BT, Jack CR Jr, Kawas CH, Klunk WE, Koroshetz WJ, Manly JJ, Mayeux R et al (2011) The diagnosis of dementia due to Alzheimer's disease: recommendations from the National Institute on AgingAlzheimer's Association workgroups on diagnostic guidelines for Alzheimer's disease. Alzheimers Dement 7:263-269. https:// doi.org/10.1016/j.jalz.2011.03.005

41. Mielke MM, Hagen CE, Xu J, Chai X, Vemuri P, Lowe VJ, Airey DC, Knopman DS, Roberts RO, Machulda MM et al (2018) Plasma phospho-tau181 increases with Alzheimer's disease clinical severity and is associated with tau- and amyloid-positron emission tomography. Alzheimer's Dement 14:989-997. https:// doi.org/10.1016/j.jalz.2018.02.013

42. Mila-Aloma M, Salvado G, Gispert JD, Vilor-Tejedor N, GrauRivera O, Sala-Vila A, Sanchez-Benavides G, Arenaza-Urquijo EM, Crous-Bou M, Gonzalez-de-Echavarri JM et al (2020) Amyloid beta, tau, synaptic, neurodegeneration, and glial biomarkers in the preclinical stage of the Alzheimer's continuum. Alzheimer's Dement 16:1358-1371. https://doi.org/10.1002/alz.12131

43. Molinuevo JL, Ayton S, Batrla R, Bednar MM, Bittner T, Cummings J, Fagan AM, Hampel H, Mielke MM, Mikulskis A et al (2018) Current state of Alzheimer's fluid biomarkers. Acta Neuropathol 136:821-853. https://doi.org/10.1007/s00401-018-1932-x 
44. Moscoso A, Grothe MJ, Ashton NJ, Karikari TK, Rodriguez JL, Snellman A, Suárez-Calvet M, Zetterberg H, Blennow K, Schöll M, Alzheimer's Disease Neuroimaging Initiative (2020) Time course of phosphorylated-tau181 in blood across the Alzheimer's disease spectrum. Brain. https://doi.org/10.1093/brain/awaa399

45. Nakamura A, Kaneko N, Villemagne VL, Kato T, Doecke J, Dore V, Fowler C, Li QX, Martins R, Rowe C et al (2018) High performance plasma amyloid-beta biomarkers for Alzheimer's disease. Nature 554:249-254. https://doi.org/10.1038/nature25456

46. Olsson B, Lautner R, Andreasson U, Ohrfelt A, Portelius E, Bjerke M, Holtta M, Rosen C, Olsson C, Strobel G et al (2016) CSF and blood biomarkers for the diagnosis of Alzheimer's disease: a systematic review and meta-analysis. Lancet Neurol 15:673-684. https://doi.org/10.1016/S1474-4422(16)00070-3

47. Orfanidis SJ (1995) Introduction to signal processing. PrenticeHall Inc, New York City

48. Palmqvist S, Janelidze S, Quiroz YT, Zetterberg H, Lopera F, Stomrud E, Su Y, Chen Y, Serrano GE, Leuzy A et al (2020) Discriminative Accuracy of Plasma Phospho-tau217 for Alzheimer Disease vs Other Neurodegenerative Disorders. JAMA 324:772-781. https://doi.org/10.1001/jama.2020.12134

49. Palmqvist S, Scholl M, Strandberg O, Mattsson N, Stomrud E, Zetterberg H, Blennow K, Landau S, Jagust W, Hansson O (2017) Earliest accumulation of beta-amyloid occurs within the defaultmode network and concurrently affects brain connectivity. Nat Commun 8:1214. https://doi.org/10.1038/s41467-017-01150-x

50. Pascoal TA, Shin M, Kang MS, Chamoun M, Chartrand D, Mathotaarachchi S, Bennacef I, Therriault J, Ng KP, Hopewell $\mathrm{R}$ et al (2018) In vivo quantification of neurofibrillary tangles with [(18)F]MK-6240. Alzheimers Res Ther 10:74. https://doi. org/10.1186/s13195-018-0402-y

51. Pascoal TA, Therriault J, Benedet AL, Savard M, Lussier FZ, Chamoun M, Tissot C, Qureshi MNI, Kang MS, Mathotaarachchi $S$ et al (2020) 18F-MK-6240 PET for early and late detection of neurofibrillary tangles. Brain. https://doi.org/10.1093/brain/awaa1 80

52. Rabinovici GD, Gatsonis C, Apgar C, Chaudhary K, Gareen I, Hanna L, Hendrix J, Hillner BE, Olson C, Lesman-Segev OH et al (2019) Association of amyloid positron emission tomography with subsequent change in clinical management among medicare beneficiaries with mild cognitive impairment or dementia. JAMA 321:1286-1294. https://doi.org/10.1001/jama.2019.2000

53. Santos JRF, Bauer C, Schuchhardt J, Wedekind D, Waniek K, Lachmann I, Wiltfang J, Vogelgsang J (2019) Validation of a prototype tau Thr231 phosphorylation CSF ELISA as a potential biomarker for Alzheimer's disease. J Neural Transm (Vienna) 126:339-348. https://doi.org/10.1007/s00702-019-01982-5

54. Sato C, Barthelemy NR, Mawuenyega KG, Patterson BW, Gordon BA, Jockel-Balsarotti J, Sullivan M, Crisp MJ, Kasten T, Kirmess KM et al (2018) Tau kinetics in neurons and the human central nervous system. Neuron 97(1284-1298):e1287. https:// doi.org/10.1016/j.neuron.2018.02.015

55. Savastano A, Flores D, Kadavath H, Biernat J, Mandelkow E, Zweckstetter M (2020) Disease-associated tau phosphorylation hinders tubulin assembly within tau condensates. Angew Chem Int Ed Engl. https://doi.org/10.1002/anie.202011157

56. Schindler SE, Bollinger JG, Ovod V, Mawuenyega KG, Li Y, Gordon BA, Holtzman DM, Morris JC, Benzinger TLS, Xiong C et al (2019) High-precision plasma beta-amyloid 42/40 predicts current and future brain amyloidosis. Neurology 93:e1647-e1659. https ://doi.org/10.1212/WNL.0000000000008081

57. Simrén J, Leuzy A, Karikari TK, Hye A, Benedet AL, LanteroRodriguez J, Mattsson-Carlgren N, Schöll M, Mecocci P, Vellas B et al (2021) The diagnostic and prognostic capabilities of plasma biomarkers in Alzheimer's disease. Alzheimers Dement. https:// doi.org/10.1002/alz.12283

58. Suarez-Calvet M, Karikari TK, Ashton NJ, Lantero Rodriguez J, Mila-Aloma M, Gispert JD, Salvado G, Minguillon C, Fauria K, Shekari M et al (2020) Novel tau biomarkers phosphorylated at T181, T217 or T231 rise in the initial stages of the preclinical Alzheimer's continuum when only subtle changes in Abeta pathology are detected. EMBO Mol Med. https://doi.org/10.15252 /emmm.202012921

59. Therriault J, Benedet A, Pascoal TA, Savard M, Ashton N, Chamoun M, Tissot C, Lussier F, Kang MSP, Bezgin G et al (2020) Determining Amyloid-beta positivity using [(18)F]AZD4694 PET imaging. J Nucl Med. https://doi.org/10.2967/jnumed.120.24520 9

60. Thijssen EH, La Joie R, Wolf A, Strom A, Wang P, Iaccarino L, Bourakova V, Cobigo Y, Heuer H, Spina S et al (2020) Diagnostic value of plasma phosphorylated tau181 in Alzheimer's disease and frontotemporal lobar degeneration. Nat Med 26:387-397. https://doi.org/10.1038/s41591-020-0762-2

61. Tolar M, Abushakra S, Hey JA, Porsteinsson A, Sabbagh M (2020) Aducanumab, gantenerumab, BAN2401, and ALZ-801the first wave of amyloid-targeting drugs for Alzheimer's disease with potential for near term approval. Alzheimers Res Ther 12:95. https://doi.org/10.1186/s13195-020-00663-w

62. Toledo JB, Xie SX, Trojanowski JQ, Shaw LM (2013) Longitudinal change in CSF Tau and Abeta biomarkers for up to 48 months in ADNI. Acta Neuropathol 126:659-670. https://doi.org/10.1007/ s00401-013-1151-4

63. Uhlmann RE, Rother C, Rasmussen J, Schelle J, Bergmann C, Ullrich Gavilanes EM, Fritschi SK, Buehler A, Baumann F, Skodras A et al (2020) Acute targeting of pre-amyloid seeds in transgenic mice reduces Alzheimer-like pathology later in life. Doi, Nat Neurosci. https://doi.org/10.1038/s41593-020-00737-w

64. Wihersaari L, Ashton NJ, Reinikainen M, Jakkula P, Pettila V, Hastbacka J, Tiainen M, Loisa P, Friberg H, Cronberg T et al (2020) Neurofilament light as an outcome predictor after cardiac arrest: a post hoc analysis of the COMACARE trial. Intensive Care Med. https://doi.org/10.1007/s00134-020-06218-9

Publisher's Note Springer Nature remains neutral with regard to jurisdictional claims in published maps and institutional affiliations. 


\section{Authors and Affiliations}

\section{Nicholas J. Ashton ${ }^{1,2,3,4}$ (D) Tharick A. Pascoal ${ }^{5,6}$ - Thomas K. Karikari ${ }^{1}$. Andréa L. Benedet ${ }^{1,5}$. Juan Lantero-Rodriguez ${ }^{1}$ - Gunnar Brinkmalm ${ }^{1}$ • Anniina Snellman ${ }^{1}$ - Michael Schöll ${ }^{1,2,10}$ • Claire Troakes ${ }^{14}$. Abdul Hye $^{3,4}$ - Serge Gauthier ${ }^{7}$. Eugeen Vanmechelen ${ }^{8} \cdot$ Henrik Zetterberg $^{1,9,10,11} \cdot$ Pedro Rosa-Neto ${ }^{1,12,13}$. Kaj Blennow ${ }^{1,9}$}

1 Department of Psychiatry and Neurochemistry, Institute of Neuroscience and Physiology, The Sahlgrenska Academy at the University of Gothenburg, Mölndal, Sweden

2 Wallenberg Centre for Molecular and Translational Medicine, University of Gothenburg, Gothenburg, Sweden

3 King's College London, Institute of Psychiatry, Psychology and Neuroscience, Maurice Wohl Institute Clinical Neuroscience Institute, London, UK

4 NIHR Biomedical Research Centre for Mental Health and Biomedical Research Unit for Dementia at South London and Maudsley NHS Foundation, London, UK

5 Translational Neuroimaging Laboratory, McGill Centre for Studies in Aging, McGill University, Montreal, QC, Canada

6 Department of Psychiatry and Neurology, University of Pittsburgh, Pittsburgh, PA, USA

7 Alzheimer's Disease Research Unit, The McGill University Research Centre for Studies in Aging, Montreal, McGill University, Montreal, QC, Canada
8 ADx NeuroSciences, Technologiepark 94, 9052 Ghent, Belgium

9 Clinical Neurochemistry Laboratory, Sahlgrenska University Hospital, Mölndal, Sweden

10 Department of Neurodegenerative Disease, UCL Institute of Neurology, Queen Square, London, UK

11 UK Dementia Research Institute at UCL, London, UK

12 Montreal Neurological Institute, Montreal, QC, Canada

13 Department of Neurology and Neurosurgery, McGill University, Montreal, QC, Canada

14 Department of Basic and Clinical Neuroscience, Institute of Psychiatry, Psychology and Neuroscience, King's College London, London, UK 